\title{
STOCHASTIC DIFFERENTIAL EQUATIONS DRIVEN BY PROCESSES GENERATED BY DIVERGENCE FORM OPERATORS I: A WONG-ZAKAI THEOREM*
}

\author{
Antoine LeJAY ${ }^{1}$
}

\begin{abstract}
We show in this article how the theory of "rough paths" allows us to construct solutions of differential equations (SDEs) driven by processes generated by divergence-form operators. For that, we use approximations of the trajectories of the stochastic process by piecewise smooth paths. A result of type Wong-Zakai follows immediately.
\end{abstract}

Mathematics Subject Classification. 60H10, 60J60.

Received April 05, 2005. Revised May 12, 2006.

\section{INTRODUCTION}

The goal of this article is to define a stochastic differential equation of the type

$$
\mathrm{d} Y_{t}=f\left(Y_{t}\right) \mathrm{d} X_{t}
$$

for a stochastic process $X$ generated by the divergence form operator

$$
L=\sum_{i=1, j}^{N} \frac{1}{2} \frac{\partial}{\partial x_{i}}\left(a_{i, j} \frac{\partial}{\partial x_{j}}\right)+\sum_{i=1}^{N} b_{i} \frac{\partial}{\partial x_{i}}
$$

where $a(x)=\left(a_{i, j}(x)\right)_{i, j=1, \ldots, N}$ is symmetric, measurable, uniformly elliptic and bounded, and $b$ is measurable and bounded. The coefficients $a$ and $b$ are not assumed to be regular.

If $a$ is smooth, then $L$ may be transformed into a non-divergence form operator

$$
\sum_{i, j=1}^{N} \frac{1}{2} a_{i, j} \frac{\partial^{2}}{\partial x_{i} \partial x_{j}}+\sum_{i, j=1}^{N} \frac{1}{2} \frac{\partial a_{i, j}}{\partial x_{i}} \frac{\partial}{\partial x_{j}}+\sum_{i=1}^{N} b_{i} \frac{\partial}{\partial x_{i}},
$$

Keywords and phrases. Rough paths, stochastic differential equations, stochastic process generated by divergence-form operators, Dirichlet process, approximation of trajectories.

* This work has been partially supported by European Union's TMR Stochastic Analysis Network (grant reference 960075).

${ }^{1}$ Projet OMEGA (INRIA Lorraine), IECN, Campus scientifique, BP 239, 54506 Vandouvre-lès-Nancy Cedex, France; Antoine.Lejay@iecn.u-nancy.fr

(c) EDP Sciences, SMAI 2006 
and in this case, $X$ is a semi-martingale. So, $Y$ may be defined as the solution of some SDE in the Itô or Stratonovich sense. But if $a$ is discontinuous, then $X$ is no longer a semi-martingale, but a Dirichlet process, that is the sum of a local martingale and a term of zero quadratic variation. The Itô theory of integration is no longer valid in this case.

However, using time-reversal techniques, stochastic integrals of the form $\int_{0}^{t} f\left(X_{s}\right) \circ \mathrm{d} \varphi\left(X_{s}\right)$ were defined by Rozkosz in [30] and Lyons and Stoica in [23]. There are also other approaches to define some integrals with respect to a Dirichlet process: see $[8,11]$ for example. But passing from integration of one forms to integration of (stochastic) differential equations has, at the best of our knowledge, not been treated.

The theory of rough paths developed by Lyons [24] (see also [17,22]) gives a pathwise view of stochastic differential equations of type (1) by viewing them as ordinary differential equations controlled by irregular paths. The price to pay, when one deals with a process $X$ of finite $\alpha$-variation, is to use the topology induced by the $\alpha$-variation norm, and to know not only the process $X$, but also its iterated integrals up to order $\lfloor\alpha\rfloor$. Thus, we need to define a path $\mathbf{X}$, called a rough path, that lives in a space of dimension $N+N^{2}+\ldots+N^{\lfloor\alpha\rfloor}$. This theory has been applied for a wide class of processes: infinite dimensional Gaussian processes [21], Lévy processes [34], fractional Brownian motion [5], Brownian Motion on fractals [13], ...

In [2], Bass, Hambly and Lyons have adapted the theory of rough paths to reversible Markov processes. This case includes processes generated by a divergence form operator when $b=0$, but their proof requires the use of the invariant measure $\int m(\mathrm{~d} x) \mathbb{P}_{x}$. Dealing with the measure $\mathbb{P}_{x}$ for an arbitrary starting point $x \in \mathbb{R}^{N}$ was left as an open problem: See Point 3 in Section 6.3 in [2]. As processes generated by divergence form operators are of finite $\alpha$-variation as soon as $\alpha>2$, we need to consider their second-order iterated integrals. The results of $[23,30]$ may be used to construct second-order iterated integrals of processes generated by divergence form operator under $\mathbb{P}_{x}$ for any $x \in \mathbb{R}^{d}$, yet their $\alpha / 2$-variations have never been studied. Moreover, the method of [2] cannot be transposed in our case, since it strongly relies on the fact that one works under the invariant measure.

In this article, we study the regularity of the second-order iterated integrals of a process generated by a divergence form operator under $\mathbb{P}_{x}$ for any $x \in \mathbb{R}^{d}$. Although a time-reversal technique plays a fundamental role here as in [2], we use it for the underlying process and not for defining the area. Thus, our proof is not an extension of the one in [2]. The regularity of the second-order iterated integrals is mainly all we need to apply the rough paths theory to this class of processes. It means that one can construct a rough path $\mathbf{X}$ lying above $X$ with $\alpha \in(2,3)$, and this construction is performed by approximating $X$ with piecewise linear functions $X^{\delta}$. Thus, the solutions $Y^{\delta}$ of the ordinary differential equations

$$
\frac{\mathrm{d} Y_{t}^{\delta}}{\mathrm{d} t}=f\left(Y_{t}^{\delta}\right) \frac{\mathrm{d} X_{t}^{\delta}}{\mathrm{d} t}, Y_{0}^{\delta}=y
$$

converge in probability under $\mathbb{P}_{x}$ for any $x$ to the solution $Y$ of (1) defined by the rough paths theory, which is denoted by

$$
\mathrm{d} Y_{t}=f\left(Y_{t}\right) \mathrm{d} \mathbf{X}_{t}, Y_{0}=y
$$

A similar result occurs when one considers stochastic integrals of type $\int_{0}^{t} g\left(X_{s}\right) \mathrm{d} \mathbf{X}_{s}$. This result is then an extension of the Wong-Zakai theorem [35].

Moreover, in our proof, we may use any deterministic family of partitions whose meshes decrease to 0 in order to construct $X^{\delta}$, while many equivalent results regarding rough paths have been proved using dyadic partitions.

In a companion article [19], we study the convergence as rough paths, or multiplicative functionals, of a family $\left(X^{\varepsilon}\right)_{\varepsilon>0}$ of processes generated by the divergence-form operators $\frac{1}{2} \frac{\partial}{\partial x_{i}}\left(a_{i, j}^{\varepsilon} \frac{\partial}{\partial x_{j}}\right)+b^{\varepsilon} \frac{\partial}{\partial x_{i}}$, when the $a^{\varepsilon}$ 's are uniformly elliptic with the same constant, and the $\left(a^{\varepsilon}, b^{\varepsilon}\right)$ 's are uniformly bounded. In particular, it will be shown that the condition UTD introduced in [6] (see also [28]) is a sufficient condition to assert that the convergence as rough paths holds and that the limit corresponds to the rough path "canonically" constructed above the limiting process $X$. 
The coefficients $a$ and $b$ may be approximated by some smooth coefficients $a^{\varepsilon}$ and $b^{\varepsilon}$ and the corresponding processes $X^{\varepsilon}$ are semi-martingales. The integrals and the stochastic differential equations, with a right choice of the rough paths $\mathbf{X}^{\varepsilon}$, correspond then to the usual Stratonovich or Itô integrals [4,32]. We will then prove that the integrals we have constructed for divergence form operators may then be approximated by usual stochastic integrals of Stratonovich or Itô type, and also correspond to the ones constructed using the time-reversal techniques in $[23,30]$.

With [19], this article provides a natural way to defined stochastic differential equations driven by processes generated by divergence form operators. In addition, the SDEs so defined possess also some natural properties: they may be approximated both by solutions of ODEs driven by piecewise smooth approximations of the processes or by semi-martingales when the coefficients of $L$ are approximated by smooth coefficients. Moreover, using a result of Wong-Zakai type, one gets immediately a change of variable formula for these kind of stochastic integrals or solutions of SDEs. And regarding stochastic integrals, we recover the objects constructed in [23,30] as a natural extension of stochastic integrals. Finally, our results may be extended to time-inhomogeneous processes or to the process $\left(\varphi_{1}(X), \ldots, \varphi_{m}(X)\right)$ where $\varphi_{i} \in \mathrm{W}_{\text {loc }}^{1, \infty}\left(\mathbb{R}^{N}\right)$ with $\nabla \varphi_{i} \in \mathrm{L}^{\infty}\left(\mathbb{R}^{N} ; \mathbb{R}^{m}\right)$ for $i=1, \ldots, m$.

Outline. In Section 2, we present the results we use on stochastic processes generated by divergence-form operators. Our main theorem is stated in Section 3. Section 3.3 contains the core of the proof, which is that piecewise linear approximations of the trajectories of $X$ and their second-order iterated integrals converge in $\alpha$-variation. In Section 4, we show how to construct different rough paths lying above the same trajectories, and their effects.

Convention. Throughout this article, we use the Einstein convention, which means that an index variable that appears twice in an expression is implicitly summed over all its possible values.

\section{ON PROCESSES GENERATED BY DIVERGENCE FORM OPERATORS}

\subsection{Construction and main properties}

We fix two constants $\lambda$ and $\Lambda$ with $0<\lambda<\Lambda$. Let $a$ be a measurable function from $\mathbb{R}^{N}$ into the space of symmetric matrices which is uniformly elliptic with respect to $\lambda$ and bounded by $\Lambda$, i.e.,

$$
\lambda|\xi|^{2} \leq a(x) \xi \cdot \xi \leq \Lambda|\xi|^{2}
$$

for any $\xi$ in $\mathbb{R}^{N}$ and almost every $x \in \mathbb{R}^{N}$, where $|\xi|$ is the Euclidean norm of $\xi$. Let also $b$ be a measurable function from $\mathbb{R}^{N}$ to $\mathbb{R}^{N}$ which is bounded by $\Lambda$. Under these conditions, the second-order differential operator

$$
L=\frac{1}{2} \frac{\partial}{\partial x_{i}}\left(a_{i, j} \frac{\partial}{\partial x_{j}}\right)+b_{i} \frac{\partial}{\partial x_{i}}
$$

with domain $\operatorname{Dom}(L)=\left\{f \in \mathrm{H}^{1}\left(\mathbb{R}^{N}\right) \mid L f \in \mathrm{L}^{2}\left(\mathbb{R}^{N}\right)\right\}$ is the infinitesimal generator of a conservative and continuous stochastic process $X$ with a density which is absolutely continuous with respect to the Lebesgue measure $[16,33]$.

If $a$ is of class $\mathcal{C}^{1}$, then $L$ may be transformed into the non-divergence form operator $\frac{1}{2} a_{i, j} \frac{\partial^{2}}{\partial x_{i} \partial x_{j}}+\frac{1}{2} \frac{\partial a_{i, j}}{\partial x_{i}} \frac{\partial}{\partial x_{j}}+$ $b_{i} \frac{\partial}{\partial x_{i}}$. If follows that $X$ is a semi-martingale and solution to some SDE. This is no longer true if the derivative of $a$ is not defined. The theory of Dirichlet forms $[10,26]$ is helpful to study the properties of such a process.

The most useful property on the transition density $p$ of $X$ is probably the Aronson estimates $[1,33]$ : there exists a constant $M$ depending only on $\lambda, \Lambda$ and the dimension $N$ such that for any $t \in(0,1]$ and any $(x, y) \in \mathbb{R}^{N} \times \mathbb{R}^{N}$

$$
\frac{1}{M t^{N / 2}} \exp \left(\frac{-M|x-y|^{2}}{t}\right) \leq p(t, x, y) \leq \frac{M}{t^{N / 2}} \exp \left(\frac{-|x-y|^{2}}{M t}\right)
$$


In other words, one may compare the transition density with the Gaussian kernel. In particular, there exists a constant $C$ that depends only on $\lambda, \Lambda$ and $N$ such that

$$
\int_{0}^{1}\left(\int_{\mathbb{R}^{N}}|p(s, x, y)|^{\alpha^{\prime}} \mathrm{d} y\right)^{\beta^{\prime} / \alpha^{\prime}} \mathrm{d} s \leq C
$$

where $\alpha^{\prime}$ and $\beta^{\prime}$ are the conjugate exponents of $\alpha \in(1, \infty]$ and $\beta \in(1, \infty]\left(\right.$ i.e., $\alpha^{\prime}=\alpha /(\alpha-1)$ and $\left.\beta^{\prime}=\beta /(\beta-1)\right)$ satisfying the relation $N / 2 \alpha+1 / \beta<1$.

In fact, $p$ also satisfies [1], Theorem 5 , p. 656

$$
\int_{0}^{1}\left(\int_{\mathbb{R}^{N}}\left(|p(s, x, y)|^{\alpha^{\prime}}+|\nabla p(s, x, y)|^{\alpha^{\prime}}\right) \mathrm{d} y\right)^{\beta^{\prime} / \alpha^{\prime}} \mathrm{d} s \leq C^{\prime}
$$

for some constant $C^{\prime}$ depending only on $\lambda, \Lambda$ and $N$, where $\alpha^{\prime}$ and $\beta^{\prime}$ are the conjugates exponents of real numbers $\alpha \in(2, \infty]$ and $\beta \in(2, \infty]$ satisfying $N / 2 \alpha+1 / \beta<1 / 2$.

Let us denote by $\left(X_{t}, \mathbb{P}_{x}, \mathcal{F}_{t} ; t \geq 0 ; x \in \mathbb{R}^{N}\right)$ the Hunt process (and then a strong Markov process) generated by $(L, \operatorname{Dom}(L))$ via the probability transition density $p$, on a probability space $(\Omega, \mathcal{F}, \mathbb{P})$. The filtration $\left(\mathcal{F}_{t}\right)_{t \geq 0}$ is the minimal admissible filtration satisfying the usual hypotheses.

Many of the constants that appear in the computations depend only on $\lambda, \Lambda, N$, and $\alpha$. This motivates the following notation.

Convention 1. An expression of type $a \preccurlyeq b$ means in fact that $a \leq C \times b$, where $C$ is a constant that depends only on $\lambda, \Lambda$, the dimension $N$, the choice of $\alpha$ and the time interval, which is $[0,1]$ here. For example, the convexity inequality $(a+b)^{\alpha} \leq 2^{\alpha-1}\left(a^{\alpha}+b^{\alpha}\right)$ is then written $(a+b)^{\alpha} \preccurlyeq a^{\alpha}+b^{\alpha}$.

An upper bound of exponential type on the probability that $X$ stays in a given ball between times 0 and $t$ follows from both the upper and lower bound in (2).

Lemma 1 (See [33], Lem. II.1.2). There exists a positive constant $C$ depending only on $\lambda, \Lambda$ and $N$ such that for any $t \in[0,1]$ and any $R \geq 0$,

$$
\mathbb{P}_{x}\left[\sup _{0 \leq s \leq t}\left|X_{s}-x\right| \geq R\right] \preccurlyeq \exp \left(\frac{-C R^{2}}{t}\right) .
$$

This lemma is useful when one has to proceed by localization. If the first-order differential term $b$ is equal to 0 , then the conclusion of Lemma 1 is true for any $t \in(0, \infty)$.

As said above, $X$ is in general not a semi-martingale, but belongs to the more general class of Dirichlet processes (the reader has to be warned that there exists several definitions of Dirichlet processes with slight differences).

We denote by $\left(\Pi^{\delta}\right)_{\delta>0}$ a family of deterministic partitions of $[0,1]$, whose meshes decrease to 0 with $\delta$.

Definition 1 (Dirichlet process along $\left.\Pi^{\delta}[9]\right)$. A stochastic process $\left(X, \mathbb{P},\left(\mathcal{F}_{t}\right)_{t \geq 0}\right)$ is called a Dirichlet process (along $\Pi^{\delta}$ ) if it admits the decomposition $X_{t}=M_{t}+A_{t}, t \in[0,1]$, where $M$ is a $\left(\mathbb{P},\left(\mathcal{F}_{t}\right)_{t \geq 0}\right)$-local martingale and $A$ is a $\left(\mathcal{F}_{t}\right)_{t \geq 0}$-adapted process such that

$$
Q(A)=\sum_{i=0}^{k^{\delta}-1}\left|A_{t_{i+1}^{\delta}}-A_{t_{i}^{\delta}}\right|^{2}
$$

converges to 0 in probability as $\delta \rightarrow 0$, where $\Pi^{\delta}$ is the partition $0 \leq t_{0}^{\delta} \leq t_{1}^{\delta} \leq \cdots \leq t_{k^{\delta}}^{\delta} \leq 1$. 
Reversing $X$ in time under $\mathbb{P}_{x}$ for a given starting point gives us more information about the term of zero quadratic variation of $X$. For that, let us denote by $\left(\overline{\mathcal{F}}_{t}\right)_{t \in[0,1]}$ the time-reversed filtration defined by

$$
\overline{\mathcal{F}}_{t}=\sigma\left(X_{s} ; t \leq s \leq 1\right) \text {. }
$$

Proposition 1 [30], Th. 2.2, p. 97; [23]). Let $\varphi$ be a function in $\mathrm{W}^{1, p}\left(\mathbb{R}^{N}\right)$ with $p>\max (2, N)$. Then, $\mathbb{P}_{x}$-a.s. for every point $x,\left(\varphi\left(X_{t}\right)\right)_{0 \leq t \leq 1}$ is a Dirichlet process with decomposition:

$$
\varphi\left(X_{t}\right)=\varphi\left(X_{0}\right)+M_{t}^{\varphi}+A_{t}^{\varphi} \text { with } A_{t}^{\varphi}=-\frac{1}{2} M_{t}^{\varphi}+\frac{1}{2}\left(\bar{M}_{1-t}^{\varphi}-\bar{M}_{1}^{\varphi}\right)+V_{t}^{\varphi}
$$

where $M^{\varphi}$ is the martingale part of $\varphi(X), \bar{M}^{\varphi}$ is the martingale part of the reversed process $\varphi\left(X_{1-t}\right)$ (this is a $\left(\overline{\mathcal{F}}_{t}\right)_{0 \leq t \leq 1}$-martingale) and

$$
\left.V_{t}^{\varphi}=\int_{0}^{t}\left(b_{i}\left(X_{s}\right) \frac{\partial \varphi}{\partial x_{i}}\left(X_{s}\right)-\frac{1}{2} p^{-1}\left(s, x, X_{s}\right) a_{i, j}\left(X_{s}\right) \frac{\partial p}{\partial x_{j}}\left(s, x, X_{s}\right)\right) \frac{\partial \varphi}{\partial x_{i}}\left(X_{s}\right)\right) \mathrm{d} s
$$

is a term of integrable variation. The martingales $M^{\varphi}$ and $\bar{M}^{\varphi}$ are square-integrable with

$$
\left\langle M^{\varphi}\right\rangle_{t}=\int_{0}^{t} a_{i, j} \frac{\partial \varphi}{\partial x_{i}} \frac{\partial \varphi}{\partial x_{j}}\left(X_{s}\right) \mathrm{d} s \text { and }\left\langle\bar{M}^{\varphi}\right\rangle_{t}=\int_{0}^{t} a_{i, j} \frac{\partial \varphi}{\partial x_{i}} \frac{\partial \varphi}{\partial x_{j}}\left(X_{1-s}\right) \mathrm{d} s .
$$

Remark 1. In [10], a process generated by a Dirichlet form may be decomposed under $\int_{\mathbb{R}^{N}} \mathrm{~d} x \mathbb{P}_{x}$ as a sum of a local martingale and a process locally of zero-energy. Definition 1 of a Dirichlet process is a more stringent.

Remark 2. If $N=1$, then Proposition 1 is also true for $p=2$ : See [29]. This proposition is also true when the coefficients $a$ and $b$ are time-inhomogeneous, as proved in [18].

Remark 3. A decomposition of type (4), which is called a Lyons-Zheng decomposition, also holds under the infinite measure $\int_{\mathbb{R}^{N}} \mathrm{~d} x \mathbb{P}_{x}$, in which case $V_{t}^{\varphi}=\int_{0}^{t} b\left(X_{s}\right) \nabla \varphi\left(X_{s}\right) \mathrm{d} s$.

Remark 4. If $\varphi \in \mathrm{W}_{\text {loc }}^{1, \infty}\left(\mathbb{R}^{N}\right)$, then (4) is still true, but $M^{\varphi}$ and $\bar{M}^{\varphi}$ are only local martingales, and $V^{\varphi}$ is not necessarily of integrable variation [30], Theorem 2.2 , p. 97 . Yet if $\nabla \varphi$ in $\mathrm{L}^{\infty}\left(\mathbb{R}^{N} ; \mathbb{R}^{N}\right)$ then it is easily checked that $M^{\varphi}$ and $\bar{M}^{\varphi}$ are still martingales, since $a$ is bounded. Besides, although $p(t, x, y)$ is singular at $t=0$, (3) implies that

$$
\mathbb{E}_{x}\left[\int_{0}^{1}\left|\mathrm{~d} V_{t}^{\varphi}\right|\right] \preccurlyeq 1
$$

\subsection{Regularity of the trajectories}

In the theory of rough paths, the results strongly depend on the regularity of the path which is considered. In our case, the regularity of a path $x:[0,1] \rightarrow \mathbb{R}^{N}$ if characterized by its $\alpha$-variation for $\alpha \geq 1$, which is defined by

$$
\operatorname{Var}_{\alpha} x=\sup _{\text {partition }\left\{t_{i}\right\}_{i=1, \ldots, k} \text { of }[0,1]}\left(\sum_{i=0}^{k-1}\left|x_{t_{i+1}}-x_{t_{i}}\right|^{\alpha}\right)^{1 / \alpha} .
$$

Let us start by recalling the Kolmogorov criterion.

Lemma 2 (Kolmogorov criterion [31], Th. 2.1 p. 25). If $(X, \mathbb{P})$ is a stochastic process on $[0,1]$ such that for some $\alpha, \varepsilon>0$,

$$
\mathbb{E}\left[\left|X_{t}-X_{s}\right|^{\alpha}\right] \leq C|t-s|^{1+\varepsilon} \text { for all } 0 \leq s \leq t \leq 1,
$$


then for any $\beta$ in $[0, \varepsilon)$,

$$
\mathbb{E}\left[\sup _{0 \leq s<t \leq 1} \frac{\left|X_{t}-X_{s}\right|^{\alpha}}{|t-s|^{\beta}}\right] \leq C^{\prime}
$$

where $C^{\prime}$ is a constant that depends only on $\alpha, \beta$ and $C$. Besides, there exists a modification of $X$ which is $\beta / \alpha$-Hölder continuous.

In particular, for any $\gamma>\alpha / \varepsilon, \mathbb{E}\left[\operatorname{Var}_{\gamma} X\right] \leq \mathbb{E}\left[\operatorname{Var}_{\gamma}(X)^{\gamma}\right]^{1 / \gamma} \leq C^{1 / \gamma}$.

The Kolmogorov criterion will be used to prove that the trajectories of the process $X$ generated by a divergence-form operator are $\beta$-Hölder continuous for any $\beta<1 / 2$, as for the trajectories of the Brownian motion. Then, almost every trajectory of $X$ is of finite $\alpha$-variation for any $\alpha>2$.

Lemma 3. Let $X$ be a process generated by a divergence form operator as in Section 2.1. For any $\alpha \geq 2$ and any $0 \leq s \leq t \leq 1$,

$$
\sup _{x \in \mathbb{R}^{N}} \mathbb{E}_{x}\left[\sup _{r \in[s, t]}\left|X_{r}-X_{s}\right|^{\alpha}\right] \preccurlyeq|t-s|^{\alpha / 2} .
$$

So, $X$ is $\beta$-Hölder continuous for any $\beta<1 / 2$, and $\mathbb{E}\left[\operatorname{Var}_{\alpha} X\right] \preccurlyeq 1$ for any $\alpha>2$.

Proof. With the Markov property,

$$
\sup _{x \in \mathbb{R}^{N}} \mathbb{E}_{x}\left[\sup _{r \in[s, t]}\left|X_{r}-X_{s}\right|^{\alpha}\right]=\sup _{x \in \mathbb{R}^{N}} \mathbb{E}_{x}\left[\sup _{r \in[0, t-s]}\left|X_{r}-x\right|^{\alpha}\right] .
$$

When $t$ and $R$ are fixed, let us denote by $\tau$ the first time $X$ exits from the ball of radius $R$ with center $x$. Then, with the help of the strong Markov property,

$$
\begin{aligned}
\sup _{x \in \mathbb{R}^{N}} \mathbb{E}_{x}\left[\sup _{r \in[0, t]}\left|X_{r}-x\right|^{\alpha}\right] & \leq R^{\alpha}+\sup _{x \in \mathbb{R}^{N}} \mathbb{E}_{x}\left[\sup _{r \in[0, t]}\left|X_{r}-x\right|^{\alpha} ; t>\tau\right] \\
& \leq R^{\alpha}+2^{\alpha-1}\left(R^{\alpha}+\sup _{x \in \mathbb{R}^{N}} \mathbb{E}_{x}\left[\sup _{r \in[\tau, t]}\left|X_{r}-X_{\tau}\right|^{\alpha} ; t>\tau\right]\right) \\
& \leq\left(2^{\alpha-1}+1\right) R^{\alpha}+2^{\alpha-1} \sup _{x \in \mathbb{R}^{N}} \mathbb{E}_{x}\left[\sup _{r \in[0, t]}\left|X_{r}-x\right|^{\alpha}\right] \sup _{x \in \mathbb{R}^{N}} \mathbb{P}_{x}[t>\tau] .
\end{aligned}
$$

But we have seen in Lemma 1 that $\sup _{x \in \mathbb{R}^{N}} \mathbb{P}_{x}[t>\tau] \leq C^{\prime} \exp \left(-C R^{2} / t\right)$, where $C$ and $C^{\prime}$ are some constants depending only on $\lambda, \Lambda$ and $N$. Hence, the lemma is proved if $R$ is chosen to be equal to $\kappa \sqrt{t}$ with $\kappa$ large enough so that $2^{\alpha-1} C^{\prime} \exp \left(-C \kappa^{2}\right) \leq 1 / 2$.

\subsection{Stochastic integrals driven by processes generated by divergence-form operators}

If $X$ denotes a process generated by a divergence form operator as in Section 2.1, Proposition 1 gives a decomposition of $X$ in term of forward and backward martingales, and a term of finite variation. In [28] (however, see Rem. 2.6 in [30]) and in [23], the decomposition (4) is used to prove existence of stochastic integrals of $f\left(X_{t}\right)$ against $\varphi\left(X_{t}\right)$ for two functions $f$ and $\varphi$.

Let $\left(\Pi^{\delta}\right)_{\delta>0}$ be a family of deterministic partitions whose mesh decreases to 0 as $\delta$ goes to 0 . For a $\delta>0$, we set $\Pi^{\delta}=\left\{0 \leq t_{1}^{\delta} \leq \cdots \leq t_{k^{\delta}}^{\delta} \leq 1\right\}$ and for $t \in[0,1], M^{\delta}(t)=i$ with $t_{i}^{\delta} \leq t<t_{i+1}^{\delta}$. 
Theorem 1 [23,28]. Let $\varphi$ and $f$ be two functions in $\mathrm{W}_{\text {loc }}^{1, p}\left(\mathbb{R}^{N}\right)$ for some $p>2 \vee N$. Then, for any starting point $x \in \mathbb{R}^{N}$ and any $t \in[0,1]$, the limits in probability under $\mathbb{P}_{x}$ of

$$
\begin{gathered}
\sum_{i=1}^{M^{\delta}(t)} f\left(X_{t_{i}^{\delta}}\right)\left(\varphi\left(X_{t_{i+1}^{\delta}}\right)-\varphi\left(X_{t_{i}^{\delta}}\right)\right) \\
\text { and } \sum_{i=1}^{M^{\delta}(t)} \frac{1}{2}\left(f\left(X_{t_{i+1}^{\delta}}\right)+f\left(X_{t_{i}^{\delta}}\right)\right)\left(\varphi\left(X_{t_{i+1}^{\delta}}\right)-\varphi\left(X_{t_{i}^{\delta}}\right)\right)
\end{gathered}
$$

exist when $\delta$ decreases to 0 . These limits are denoted by $\int_{0}^{t} f\left(X_{s}\right) \mathrm{d} \varphi\left(X_{s}\right)$ for (8) and $\int_{0}^{t} f\left(X_{s}\right) \circ \mathrm{d} \varphi\left(X_{s}\right)$ for (9).

The theory of rough paths requires one to construct the integral of $X$ against itself, which can be done using this Theorem. Then, one may consider both stochastic differential equations controlled by $X$ and integrals driven by $X$. In the companion paper [18], we will identify the latter integrals with the one constructed in Theorem 1 .

Using Theorem 1, we define for $i, j=1, \ldots, N$ and $t \in[0,1]$,

$$
\begin{gathered}
\int_{0}^{t} X_{s}^{i} \mathrm{~d} X_{s}^{j}=\lim _{\delta \rightarrow 0} \sum_{k=1}^{M^{\delta}(t)} X_{t_{k}^{\delta}}^{i}\left(X_{t_{k+1}^{\delta}}^{j}-X_{t_{k}^{\delta}}^{j}\right), \\
\text { and } \int_{0}^{t} X_{s}^{i} \circ \mathrm{d} X_{s}^{j}=\lim _{\delta \rightarrow 0} \frac{1}{2} \sum_{k=1}^{M^{\delta}(t)}\left(X_{t_{k}^{\delta}}^{i}+X_{t_{k+1}^{\delta}}^{i}\right)\left(X_{t_{k+1}^{\delta}}^{j}-X_{t_{k}^{\delta}}^{j}\right),
\end{gathered}
$$

where the convergences hold in probability under $\mathbb{P}_{x}, x \in \mathbb{R}^{N}$.

Let us end this section by showing the relationship between the two integrals. For $t \in(0,1]$, let us also set

$$
\begin{aligned}
& Q_{t}^{\delta, i, j}=\frac{1}{2} \frac{t-t_{M^{\delta}(t)}^{\delta}}{t_{M^{\delta}(t)+1}^{\delta}-t_{M^{\delta}(t)}^{\delta}}\left(X_{t_{M^{\delta}(t)}^{i}+1}^{i}-X_{t_{M^{\delta}(t)}^{i}}^{i}\right)\left(X_{t_{M^{\delta}(t)}^{j}+1}^{j}-X_{t_{M^{\delta}(t)}^{j}}^{j}\right) \\
& +\frac{1}{2} \sum_{k=0}^{M^{\delta}(t)-1}\left(X_{t_{k}^{\delta}+1}^{i}-X_{t_{k}^{\delta}}^{i}\right)\left(X_{t_{k}^{\delta}+1}^{j}-X_{t_{k}^{\delta}}^{j}\right) \text {. }
\end{aligned}
$$

Proposition 2 [28]. For any $x \in \mathbb{R}^{N}$ and $t \in[0,1], Q_{t}^{\delta, i, j}$ converges in probability under $\mathbb{P}_{x}$ to $\frac{1}{2}\left\langle M^{i}, M^{j}\right\rangle_{t}$ when $\delta \rightarrow 0$, where $M$ is the martingale part of $X$.

It follows easily that

$$
\int_{0}^{t} X_{s}^{i} \circ \mathrm{d} X_{s}^{j}=\int_{0}^{t} X_{s}^{i} \mathrm{~d} X_{s}^{j}+\frac{1}{2}\left\langle M^{i}, M^{j}\right\rangle_{t}=\int_{0}^{t} X_{s}^{i} \mathrm{~d} X_{s}^{j}+\frac{1}{2} \int_{0}^{t} a_{i, j}\left(X_{s}\right) \mathrm{d} s
$$

as for the semi-martingale case. Note that because $X$ is a Dirichlet process, one may define the brackets of $X$ as equal to the brackets of its martingale part $M$.

\section{Rough Paths AND PROCESSES Generated by DiVERGENCE FORM OPERATORS}

The theory of rough paths allows us to construct integrals and solutions of differential equations driven by an irregular path, provided that this path is "enhanced" with another one that plays the role of the iterated integrals. In this section, we show how to construct a rough path $\mathbf{X}$ lying above $X$ by proving that the secondorder iterated integrals of $X$ (since $X$ is of finite $\alpha$-variation with $\alpha \in(2,3]$ ) have the required regularity. 
Before stating our main result in Theorem 2, we recall a few definitions. The reader is referred to $[17,22,24]$ for some expositions of this theory.

\subsection{A few definitions from the theory of rough paths}

We recall that from the rough paths terminology, a rough path, or a multiplicative functional, (of order 2) is a function $\mathbf{X}=\left(\mathbf{X}^{1}, \mathbf{X}^{2}\right)$ defined from $\Delta_{+}=\{(s, t) \mid 0 \leq s \leq t \leq 1\}$ to $\mathbb{R}^{N} \times\left(\mathbb{R}^{N} \otimes \mathbb{R}^{N}\right)$ such that for some function $X:[0, T] \rightarrow \mathbb{R}^{N}$,

$$
\mathbf{X}_{s, t}^{i, 1}=X_{t}^{i}-X_{s}^{i} \text { and } \mathbf{X}_{s, t}^{i, j, 2}=\mathbf{X}_{s, r}^{i, j, 2}+\mathbf{X}_{r, t}^{i, j, 2}+\mathbf{X}_{s, r}^{i, 1} \times \mathbf{X}_{r, t}^{j, 1}
$$

for all $0 \leq s \leq r \leq t \leq 1$.

The function $\mathbf{X}$ is a smooth rough path if $t \mapsto \mathbf{X}_{0, t}$ is also smooth.

The function $\mathbf{X}$ is a geometric rough path if there exists a function $\mathbf{Y}$ from $\Delta_{+}$into $\mathbb{R}^{N} \otimes \mathbb{R}^{N}$ such that

$$
\begin{gathered}
\mathbf{Y}_{s, t}^{i, j}=-\mathbf{Y}^{j, i} \text { if } i \neq j \text { and } \mathbf{Y}^{i, i}=0 \\
\mathbf{Y}_{s, t}^{i, j}=\mathbf{Y}_{s, r}^{i, j}+\mathbf{Y}_{r, t}^{i, j}+\frac{1}{2}\left(\mathbf{X}_{s, r}^{i, 1} \times \mathbf{X}_{r, t}^{j, 2}-\mathbf{X}_{s, r}^{j, 1} \times \mathbf{X}_{r, t}^{i, 2}\right), \\
\mathbf{X}_{s, t}^{i, j, 2}=\mathbf{Y}_{s, t}^{i, j}+\frac{1}{2} \mathbf{X}_{s, t}^{i, 1} \times \mathbf{X}_{s, t}^{j, 1} .
\end{gathered}
$$

We have to note that if $\mathbf{X}$ is a geometric rough path and if $\mathbf{Z}:[0,1] \rightarrow \mathbb{R}^{N} \otimes \mathbb{R}^{N}$ satisfies (12), then $\left(\mathbf{X}_{s, t}+\right.$ $\left.\mathbf{Z}_{t}-\mathbf{Z}_{s}\right)_{(s, t) \in \Delta_{+}}$is also a geometric rough path: see Section 4.1. Of course, any geometric rough path is also a rough path, but the converse is not true: see [25] for more details. This distinction will be important here, since we will construct two integrals, one of Stratonovich type and the other of Itô type. The first construction uses indeed geometric rough paths while constructing integrals of Itô types requires one to control also the brackets of the process.

We will not use the algebraic characterization of a geometric rough path, but another one that identifies them with the limit of a sequence of smooth rough paths. But first, we introduce the $\alpha$-variation of $\mathbf{X}$.

If $X: \Delta_{+} \rightarrow \mathrm{V}$, where $\mathrm{V}$ is a Banach space with a norm $|\cdot|$, then the $\alpha$-variation of $X$ is

$$
\operatorname{Var}_{\alpha} X=\sup _{\text {partition }\left\{t_{i}\right\}_{i=1, \ldots, k} \text { of }[0,1]}\left(\sum_{i=0}^{k-1}\left|X_{t_{i}, t_{i+1}}\right|^{\alpha}\right)^{1 / \alpha}
$$

provided that this quantity is finite. If $X:[0,1] \rightarrow \mathrm{V}$, then its $\alpha$-variation is defined by (13), where $X_{s, t}$ is replaced by $X_{t}-X_{s}$, so that we recover (6).

For $\alpha \geq 2$, we denote by $\mathcal{V}^{\alpha}$ the space of rough paths $\mathbf{X}=\left(\mathbf{X}^{1}, \mathbf{X}^{2}\right)$ such that $\mathbf{X}$ is continuous, $\mathbf{X}^{1}$ is of finite $\alpha$-variation, and $\mathbf{X}^{2}$ is of finite $\alpha / 2$-variation. This space is equipped with the norm

$$
\|\mathbf{X}\|_{\mathcal{V}^{\alpha}}=\|\mathbf{X}\|_{\infty}+\operatorname{Var}_{\alpha}\left(\mathbf{X}^{1}\right)+\operatorname{Var}_{\alpha / 2}\left(\mathbf{X}^{2}\right) .
$$

One can easily show that $\mathcal{V}^{\alpha} \subset \mathcal{V}^{\alpha^{\prime}}$ for all $\alpha^{\prime} \leq \alpha$.

Proposition 3 [12,22,24]. A rough path $\mathbf{X}: \Delta_{+} \rightarrow \mathbb{R}^{N} \times\left(\mathbb{R}^{N} \otimes \mathbb{R}^{N}\right)$ in $\mathcal{V}^{\alpha}, \alpha \geq 2$ is a geometric one if there exists a sequence of functions $X^{\delta}:[0,1] \rightarrow \mathbb{R}^{N}$ such that $\mathbf{X}^{\delta}$ converges uniformly to $\mathbf{X}$ and in $\mathcal{V}^{\alpha^{\prime}}$ for all $\alpha^{\prime}>\alpha$, where

$$
\mathbf{X}_{s, t}^{1}=X_{t}^{\delta}-X_{s}^{\delta} \text { and } \mathbf{X}_{s, t}^{i, j, 2, \delta}=\int_{s}^{t}\left(X_{r}^{i, \delta}-X_{s}^{i, \delta}\right) \mathrm{d} X_{r}^{j, \delta} .
$$

In other words, the geometric rough paths are the one that can be approximated by a sequence of smooth rough paths $\mathbf{X}^{\delta}$ whose second-order term $\mathbf{X}^{2, \delta}$ is constructed using the iterated integrals of $X^{\delta}$. Of course, $\mathbf{X}^{\delta}$ is a also a geometric rough path for any $\delta>0$. 


\subsection{The main result}

With the help of Theorem 1 , let us define for $i, j \in\{1, \ldots, N\}$ and $(s, t) \in \Delta_{+}$,

$$
K_{s, t}^{i, j}(X)=\int_{s}^{t}\left(X_{r}^{i}-X_{s}^{i}\right) \circ \mathrm{d} X_{r}^{j} \text { and } K_{s, t}^{i, j, \mathrm{ito}}(X)=\int_{s}^{t}\left(X_{r}^{i}-X_{s}^{i}\right) \mathrm{d} X_{r}^{j} .
$$

We denote by $K(X)$ and $K^{\text {itô }}(X)$ the families $\left(K_{s, t}^{i, j}(X)\right)_{i, j \in\{1, \ldots, N\},(s, t) \in \Delta_{+}}$and $\left(K_{s, t}^{i, j, \text { ito }}(X)\right)_{i, j \in\{1, \ldots, N\},(s, t) \in \Delta_{+}}$. We also set $X_{s, t}=X_{t}-X_{s}$. It is easily verified that $\left(X_{s, t}, K_{s, t}(X)\right)_{(s, t) \in \Delta_{+}}$and $\left(X_{s, t}, K_{s, t}^{\text {itô }}(X)\right)_{(s, t) \in \Delta_{+}}$are rough paths, in the sense they satisfy (11).

Given $\omega$ in the probability space $\Omega$, let $X^{\delta}(\omega)$ be the piecewise linear approximation of $X(\omega)$ along $\Pi^{\delta}$, that is

$$
X_{t}^{\delta}(\omega)=X_{t_{i}}(\omega)+\frac{t-t_{i}}{t_{i+1}-t_{i}}\left(X_{s_{i+1}}(\omega)-X_{t_{i}}(\omega)\right) \text { for } t \in\left[t_{i}, t_{i+1}\right] .
$$

For $i, j=1, \ldots, N$ and $0 \leq s \leq t \leq 1$, we also denote by $K_{s, t}^{i, j}\left(X^{\delta}\right)$ the ordinary integrals

$$
K_{s, t}^{i, j}\left(X^{\delta}\right)=\int_{s}^{t}\left(X_{r}^{i, \delta}-X_{s}^{i, \delta}\right) \mathrm{d} X_{r}^{j, \delta} \text { and } K_{s, t}^{i, j, \mathrm{ito}}\left(X^{\delta}\right)=K_{s, t}\left(X^{\delta}\right)-\left(Q_{t}^{i, j, \delta}-Q_{s}^{i, j, \delta}\right),
$$

where $Q^{\delta}$ have been defined by (10). We denote by $K\left(X^{\delta}\right)$ and $K^{\text {ito }}\left(X^{\delta}\right)$ the families $\left.\left(K_{s, t}^{i, j}\left(X^{\delta}\right)\right)_{i, j \in\{1, \ldots, N}\right\},(s, t) \in \Delta_{+}$ and $\left(K_{s, t}^{\mathrm{ito}, i, j}\left(X^{\delta}\right)\right)_{i, j \in\{1, \ldots, N\},(s, t) \in \Delta_{+}}$.

Our main result is the following.

\section{Theorem 2.}

(i) The rough paths $(X, K(X))$ and $\left(X, K^{\mathrm{ito}}(X)\right)$ are of finite $\alpha$-variation for any $\alpha>2$.

(ii) For any $\alpha>2$, the sequences of smooth rough paths $\left(X^{\delta}, K\left(X^{\delta}\right)\right)_{\delta>0}$ and $\left(X^{\delta}, K^{\mathrm{itô}}\left(X^{\delta}\right)\right)_{\delta>0}$ converge in probability in $\mathcal{V}^{\alpha}$ respectively to $(X, K(X))$ and $\left(X, K^{\text {itô }}(X)\right)$.

(iii) The rough path $(X, K(X))$ is a geometric one.

In this theorem, we give only a convergence in probability, while there are other types of processes (Brownian motion [21], fractional Brownian motion [5], semi-martingales [4], ...) for which an almost sure convergence is given. But we deal with a family of general, deterministic partitions, and not with dyadic partitions.

The following corollaries are then immediate using the results from the theory of rough paths [17, 22,24].

We set $\mathbf{X}=\left(X_{s, t}, K_{s, t}(X)\right)_{(s, t) \in \Delta_{+}}$and $\mathbf{X}^{\mathrm{itô}}=\left(X_{s, t}, K_{s, t}^{\mathrm{ito}}(X)\right)_{(s, t) \in \Delta_{+}}$.

Corollary 1. Let $f=\left(f^{1}, \ldots, f^{N}\right)$ be a function such that: For $i=1, \ldots, N, f^{i}$ is a continuous function from $\mathbb{R}^{N}$ to $\mathbb{R}^{m}$ with a derivative which is $\gamma$-Hölder continuous for some $\gamma>0$.

Then the integrals $\mathbf{Z}=\int_{0}^{\cdot} f\left(X_{s}\right) \mathrm{d} \mathbf{X}_{s}$ and $\mathbf{Z}^{\mathrm{ito}}=\int_{0}^{\cdot} f\left(X_{s}\right) \mathrm{d} \mathbf{X}_{s}^{\text {itô }}$ exist and define respectively a geometric rough path and a rough path, both of finite $\alpha$-variation for any $\alpha>2$.

Let $Z^{\delta}$ and $Z^{\delta \text { itô }}$ be the processes in $\mathbb{R}^{m}$ defined by the ordinary integrals

$$
\begin{aligned}
Z_{t}^{\delta} & =z+\int_{0}^{t} f^{i}\left(X_{r}^{\delta}\right) \mathrm{d} X_{r}^{\delta, i}, \\
\text { and } Z_{t}^{\delta, \text { ito }} & =z+\int_{0}^{t} f^{i}\left(X_{r}^{\delta}\right) \mathrm{d} X_{r}^{\delta, i}+\int_{0}^{t} \frac{\partial f^{i}}{\partial x_{k}}\left(X_{r}^{\delta}\right) \mathrm{d} Q_{r}^{\delta, i, k}
\end{aligned}
$$

where $z \in \mathbb{R}^{m}$ is fixed. Then, for any $x \in \mathbb{R}^{N}, Z^{\delta}$ and $Z^{\delta \text {,itô }}$ converge in probability under $\mathbb{P}_{x}$ to the stochastic

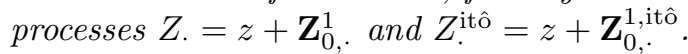

In fact, it will be shown in [19] that $Z_{t}$ is equal to the Stratonovich integral $z+\int_{0}^{t} f^{i}\left(X_{r}\right) \circ \mathrm{d} X_{r}^{i}$ defined in Theorem 1 , while $Z_{t}^{\text {itô }}$ is equal to the Itô integral $z+\int_{0}^{t} f^{i}\left(X_{r}\right) \mathrm{d} X_{r}^{i}$. 
Corollary 2. Let $g=\left(g^{1}, \ldots, g^{N}\right)$ be a function such that $g^{i}$ is a continuous function from $\mathbb{R}^{m}$ to $\mathbb{R}^{N}$ with a derivative which is $\gamma$-Hölder continuous for some $\gamma>0$.

Then there exist some solutions $\mathbf{Y}$ and $\mathbf{Y}^{\text {itô }}$ to the stochastic differential equations $\mathbf{Y}_{0, t}=\int_{0}^{t} g\left(y+\mathbf{Y}_{0, s}^{1}\right) \mathrm{d} \mathbf{X}_{s}$ and $\mathbf{Y}_{0, t}^{\mathrm{ito}}=\int_{0}^{t} g\left(y+\mathbf{Y}_{0, s}^{\mathrm{ito}, 1}\right) \mathrm{d} \mathbf{X}_{s}^{\mathrm{ito}}$. These objects $\mathbf{Y}$ and $\mathbf{Y}^{\mathrm{ito}}$ are rough paths of finite $\alpha$-variation for any $\alpha>2$ and $\mathbf{Y}$ is a geometric rough path.

If $g^{i}$ is twice differentiable and $\partial_{x_{\ell}, x_{j}}^{2} g^{i}$ is $\gamma$-Hölder continuous for some $\gamma>0$, then $\mathbf{Y}$ and $\mathbf{Y}^{\text {itô }}$ are unique.

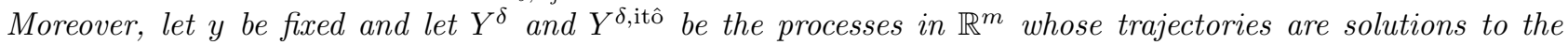
ordinary differential equations

$$
\begin{gathered}
Y_{t}^{\delta}=y+\int_{0}^{t} g^{i}\left(Y_{r}^{\delta}\right) \mathrm{d} X_{r}^{i, \delta} \\
\text { and } Y_{t}^{\ell, \delta, \text { itô }}=y+\int_{0}^{t} g_{\ell}^{i}\left(Y_{r}^{\delta, \text { itô }}\right) \mathrm{d} X_{r}^{i, \delta}+\int_{0}^{t} g_{k}^{i}\left(Y_{r}^{\delta, \text { itô }}\right) \frac{\partial g_{\ell}^{j}}{\partial x_{k}}\left(Y_{r}^{\delta, \text { itô }}\right) \mathrm{d} Q_{r}^{\delta, i, j}
\end{gathered}
$$

for $\ell=1, \ldots, m$. Then, for any $x \in \mathbb{R}^{N}, Y^{\delta}$ and $Y^{\delta \text {,itô }}$ converge in probability under $\mathbb{P}_{x}$ as $\delta \rightarrow 0$ respectively to $Y=y+\mathbf{Y}_{0, \text {, }}^{1}$ and $Y^{\text {itô }}=y+\mathbf{Y}_{0, \text {, }}^{1, \text { itô }}$.

Remark 5. Indeed, all the previous results are true if $X$ is replaced by $\varphi(X)$, where $\varphi$ is a function in $\mathrm{W}_{\text {loc }}^{1, \infty}\left(\mathbb{R}^{N} ; \mathbb{R}^{m}\right)$ with $\nabla \varphi \in \mathrm{L}^{\infty}$.

Remark 6. Using some of the results in [18], these results are also be true for inhomogeneous diffusion processes.

Remark 7. Applying the Newton formula on the change of variables to a function of class $\mathcal{C}^{2+\gamma}$ with $\gamma>0$ to $Z^{\delta}, Z^{\delta \text {,itô }}, Y^{\delta}$ and $Y^{\delta \text {,itô }}$, it is immediately established that the Stratonovich and Itô formula hold for $X$ and also for $Y, Y^{\text {itô }}, Z$ and $Z^{\text {itô }}$. The Itô formula for $X$ could be proved under weaker assumptions for $X$ (See for example $[7,10,28]$ for different approaches).

\subsection{Notations and useful results}

We recall first a useful tightness criterion on the space of rough paths [17].

Lemma 4 (A tightness criterion). Let $\left(\mathbf{X}^{\delta}\right)_{\delta>0}$ be a family of rough paths of finite $\alpha$-variation such that for any $C>0$ and for $i=1, \ldots,\lfloor\alpha\rfloor$,

$$
\begin{gathered}
\limsup _{\eta \rightarrow 0} \sup _{\delta>0} \mathbb{P}\left[\sup _{|t-s| \leq \eta}\left|\mathbf{X}_{s, t}^{i, \delta}\right|>C\right]=0 \\
\quad \lim _{\kappa \rightarrow \infty} \sup _{\delta>0} \mathbb{P}\left[\operatorname{Var}_{\alpha / i}\left(\mathbf{X}^{i, \delta}\right)>\kappa\right]=0
\end{gathered}
$$

Then, there exists a rough path $\mathbf{X}$ of finite $\alpha$-variation such that $\mathbf{X}^{\delta}$ converges in distribution to $\mathbf{X}$ in $\mathcal{V}^{\alpha^{\prime}}$ for any $\alpha^{\prime}>\alpha$.

Furthermore, if $\mathbf{X}^{\delta}$ lies above $X^{\delta}$, and $\left(X_{0}^{\delta}\right)_{\delta>0}$ is tight, then the limit $\mathbf{X}$ lies above $X_{t}=\mathbf{X}_{0, t}^{1}+X_{0}$, where $X_{0}$ is a limit in distribution of $X_{0}^{\delta}$.

Remark 8. As the space of continuous functions of finite $\alpha$-variation is not separable, the convergence in distribution in $\mathcal{V}^{\alpha^{\prime}}$ of $\mathbf{X}^{\delta}$ to $\mathbf{X}$ may not imply that (15) is true.

Convention 2. In the sequel, we choose a family of deterministic partitions $\Pi^{\delta}$ whose meshes decrease to 0 with $\delta$. When $\delta$ is fixed, then the point of the partitions are denoted by $\left\{t_{i}\right\}_{i}$ and the reference to $\delta$ is implicit.

Let $\Pi=\left\{0 \leq s_{1} \leq \cdots \leq s_{n} \leq 1\right\}$ be a partition of $[0,1]$. When $\delta$ is fixed, the expression

$$
A(s, t)=\sum_{i=0}^{\ell} B\left(t_{i}, t_{i+1}\right) \quad\left(\operatorname{resp} . \sum_{\Pi} \sum_{i=0}^{\ell} B\left(s_{j}, s_{j+1}, t_{i}, t_{i+1}\right)\right)
$$


means that the points $t_{i}$ are all the points of $\Pi^{\delta}$ such that $s<t_{1} \leq \cdots \leq t_{\ell}<t$ (resp. $\left.s_{j}<t_{1} \leq \cdots \leq t_{\ell}<s_{j+1}\right)$. We also use the convention that $t_{0}=s$ and $t_{\ell+1}=t$ (resp. $t_{0}=s_{j}$ and $t_{\ell+1}=s_{j+1}$ ). Thus, $t_{0}$ and $t_{\ell+1}$ are not necessarily in $\Pi^{\delta}$. However, we denote by $t_{-1}$ and $t_{\ell+2}$ the closest points in $\Pi^{\delta}$ to $s$ and $t$ (resp. $s_{j}$ and $s_{j+1}$ ). This convention also covers expressions like $\sum_{i=1}^{\ell-1} B\left(t_{i}, t_{i+1}\right)$, in which case the sum takes into account only the points of $\Pi^{\delta}$ in $[s, t]$.

Let us recall an important fact.

Lemma 5. If $f$ is a non-negative function and $\alpha \geq 1$, then

$$
\sum_{\Pi}\left(\int_{s_{i}}^{s_{i+1}} f(r) \mathrm{d} r\right)^{\alpha} \leq\left(\int_{0}^{1} f(r) \mathrm{d} r\right)^{\alpha} .
$$

So, if $F(s, t)=\int_{s}^{t} f(r) \mathrm{d} r$, it is immediate that $\operatorname{Var}_{\alpha} F \leq \int_{0}^{1} f(r) \mathrm{d} r$.

Proof. This inequality is a generalization of the fact that for any $\alpha>1$ and any $s \leq u \leq t$,

$$
|t-u|^{\alpha}+|u-s|^{\alpha} \leq|t-s|^{\alpha}
$$

and this lemma is easily proved.

\subsection{Piecewise approximation of the trajectories}

From now, $X$ denotes a process generated by a divergence-form operator under the hypotheses given in Section 2.1. Let $X^{\delta}$ be the piecewise linear approximation of $X$ given by (14).

Lemma 6. For any $\delta>0, \operatorname{Var}_{\alpha}\left(X^{\delta}\right) \preccurlyeq \operatorname{Var}_{\alpha}(X)$.

Proof. Let $0 \leq t_{1} \leq \cdots \leq t_{k^{\delta}} \leq 1$ be the points of the partition of $\Pi^{\delta}$. Let $\Pi=\left\{s_{i} \mid 0 \leq s_{1} \leq \cdots \leq s_{n} \leq 1\right\}$ be a partition of $[0,1]$. We introduce two sets of indices (with the convention that $t_{0}=s_{0}=0$ and $s_{n+1}=t_{k^{\delta}+1}=1$ ): For $j=0, \ldots, k^{\delta}$, we set

$$
\begin{aligned}
\Pi_{j} & =\left\{i \mid s_{i} \in\left[t_{j}, t_{j+1}\right]\right\}, \\
\Pi_{\text {left }} & =\left\{i \mid \exists j, j^{\prime} \text { s.t. } s_{i} \leq t_{j} \leq t_{j^{\prime}}<s_{i+1}\right\} .
\end{aligned}
$$

For each $j$, we remark that (with the convention that a sum over the empty set is 0 ),

$$
\sum_{i \in \Pi_{j}, i \neq \max \Pi_{j}}\left|X_{s_{i+1}}^{\delta}-X_{s_{i}}^{\delta}\right|^{\alpha} \leq \sum_{i \in \Pi_{j}, i \neq \max \Pi_{j}} \frac{\left|s_{i+1}-s_{i}\right|^{\alpha}}{\left|t_{j+1}-t_{j}\right|^{\alpha}}\left|X_{t_{j+1}}-X_{t_{j}}\right|^{\alpha} .
$$

In view of (16),

Thus,

$$
\sum_{i \in \Pi_{j}, i \neq \max \Pi_{j}}\left|s_{i+1}-s_{i}\right|^{\alpha} \leq\left|s_{\max \Pi_{j}}-s_{\min \Pi_{j}}\right|^{\alpha} \leq\left|t_{j+1}-t_{j}\right|^{\alpha}
$$

$$
\sum_{j=0}^{k^{\delta}} \sum_{i \in \Pi_{j},}\left|X_{s_{i+1}}^{\delta}-X_{s_{i}}^{\delta}\right|^{\alpha} \leq \sum_{j=0}^{k^{\delta}}\left|X_{t_{j+1}}-X_{t_{j}}\right|^{\alpha} \leq \operatorname{Var}_{\alpha}(X)^{\alpha} .
$$

For $i$ in $\Pi_{\text {left }}$, since $\left|X_{s}^{\delta}-X_{t_{j}}\right| \leq\left|X_{t_{j+1}}-X_{t_{j}}\right|$ and $\left|X_{s}^{\delta}-X_{t_{j+1}}\right| \leq\left|X_{t_{j+1}}-X_{t_{j}}\right|$ for any $s \in\left[t_{j}, t_{j+1}\right)$,

$$
\begin{aligned}
\left|X_{s_{i}}^{\delta}-X_{s_{i+1}}^{\delta}\right|^{\alpha} & \preccurlyeq\left|X_{s_{i}}^{\delta}-X_{t_{j}}\right|^{\alpha}+\left|X_{t_{j}}-X_{t_{j^{\prime}}}\right|^{\alpha}+\left|X_{t_{j^{\prime}}}-X_{s_{i+1}}^{\delta}\right|^{\alpha} \\
& \leq\left|X_{t_{j-1}}-X_{t_{j}}\right|^{\alpha}+\left|X_{t_{j}}-X_{t_{j^{\prime}}}\right|^{\alpha}+\left|X_{t_{j^{\prime}}}-X_{t_{j^{\prime}+1}}\right|^{\alpha} .
\end{aligned}
$$


Hence,

Finally, let us remark that

$$
\sum_{i \in \Pi_{\text {left }}}\left|X_{s_{i+1}}^{\delta}-X_{s_{i}}^{\delta}\right|^{\alpha} \preccurlyeq \operatorname{Var}_{\alpha}(X)^{\alpha}
$$

$$
\sum_{i=0}^{n-1}\left|X_{s_{i+1}}^{\delta}-X_{s_{i}}^{\delta}\right|^{\alpha} \leq \sum_{i \in \Pi_{\text {left }}}\left|X_{s_{i+1}}^{\delta}-X_{s_{i}}^{\delta}\right|^{\alpha}+\sum_{j=0}^{k^{\delta}} \sum_{i \in \Pi_{j} \text { s.t. } i \neq \max \Pi_{j}}\left|X_{s_{i+1}}^{\delta}-X_{s_{i}}^{\delta}\right|^{\alpha} \preccurlyeq \operatorname{Var}_{\alpha}(X)^{\alpha}
$$

and the lemma is proved.

Corollary 3. For any $\alpha>2, X^{\delta}$ converges almost surely in $\alpha$-variation to $X$.

Proof. This follows immediately from the fact that for all $\alpha^{\prime}>\alpha$,

$$
\operatorname{Var}_{\alpha^{\prime}}\left(X^{\delta}\right)^{\alpha^{\prime}} \leq\left\|X^{\delta}\right\|_{\infty}^{\left(\alpha^{\prime}-\alpha\right) / \alpha^{\prime}} \operatorname{Var}_{\alpha}\left(X^{\delta}\right)^{\alpha},
$$

the uniform convergence of $X^{\delta}$ to $X$ and Lemma 6 .

\subsection{Convergence of the iterated integrals of second order}

We now turn to the convergence of the iterated integrals. Indeed, we need to prove the convergence of the iterated integrals for all the couples of indices in $\{1, \ldots, N\}$.

For the sake of simplicity, we set $Y=X^{j}$ and $Z=X^{i}$ for $i, j \in\{1, \ldots, N\}$. Note that $i$ and $j$ may be equal. We now change our notations: we set $X=(Z, Y)$ and $X^{\delta}=\left(Z^{\delta}, Y^{\delta}\right)$, where $Y^{\delta}$ (resp. $Z^{\delta}$ ) is the piecewise linear approximation of $Y$ (resp. $Z$ ) along $\Pi^{\delta}$.

We denote by $K_{0, t}(X)$ the Stratonovich integral $K_{0, t}(X)=\int_{0}^{t}\left(Z_{r}-Z_{0}\right) \circ \mathrm{d} Y_{r}$. Moreover, we set $K_{0, t}\left(X^{\delta}\right)=$ $\int_{0}^{t}\left(Z_{r}^{\delta}-Z_{0}^{\delta}\right) \mathrm{d} Y_{r}^{\delta}$.

For any $0 \leq s \leq t \leq 1$, we define $K_{s, t}(X)$ and $K_{s, t}\left(X^{\delta}\right)$ by

$$
\begin{aligned}
K_{s, t}(X) & =K_{t}(X)-K_{s}(X)-\left(Z_{s}-Z_{0}\right)\left(Y_{t}-Y_{s}\right), \\
K_{s, t}\left(X^{\delta}\right) & =K_{t}\left(X^{\delta}\right)-K_{s}\left(X^{\delta}\right)-\left(Z_{s}^{\delta}-Z_{0}^{\delta}\right)\left(Y_{t}^{\delta}-Y_{s}^{\delta}\right) .
\end{aligned}
$$

Accordingly, $K_{s, t}(X)=\int_{s}^{t}\left(Z_{r}-Z_{s}\right) \circ \mathrm{d} Y_{r}$ and $K_{s, t}\left(X^{\delta}\right)=\int_{s}^{t}\left(Z_{r}^{\delta}-Z_{s}^{\delta}\right) \mathrm{d} Y_{r}^{\delta}$. With these notations, $\left(X_{s, t}, K_{s, t}(X)\right)_{(s, t) \in \Delta_{+}}$and $\left(X_{s, t}^{\delta}, K_{s, t}\left(X^{\delta}\right)\right)_{(s, t) \in \Delta_{+}}$become rough paths.

For an arbitrary $x$ in $\mathbb{R}^{N}$, we set $\mathbb{P}=\mathbb{P}_{x}$ and $\mathbb{E}=\mathbb{E}_{x}$.

Notation 1. For each $\delta>0$, we consider a function $K^{\delta}:(s, t) \mapsto K_{s, t}^{\delta}$ continuous on $\Delta_{+}$.

We consider the following two conditions on $\left(K^{\delta}\right)_{\delta>0}$ :

For all $\varepsilon>0, C>0$, there exists $\eta$ depending only on $\lambda, \Lambda$ and the dimension $N$ such that

$\sup _{\delta>0} \mathbb{P}\left[\sup _{\substack{0 \leq s \leq t \leq 1 \\ t-s<\eta}}\left|K_{s, t}^{\delta}\right|>C\right]<\varepsilon$,

and

For all $\varepsilon>0$ there exists $C$ that depends only on $\alpha>2, \lambda, \Lambda$ and the dimension $N$ ) such that $\sup _{\delta>0} \mathbb{P}\left[\operatorname{Var}_{\alpha / 2}\left(K^{\delta}\right)>C\right]<\varepsilon$. 
We may also consider $(\star)$ and $(\star \star)$ for a single functions $K: \Delta_{+} \rightarrow \mathbb{R}$, which means that we use it for the constant sequence $(K)_{\delta>0}$.

Note that the conditions $(\star)$ and $(\star \star)$ are stronger than the hypotheses of Lemma 4 , since the constants depend only on the parameters $\lambda, \Lambda$ and the dimension $N$. These stronger statements will be used in the companion paper [19].

Proposition 4. The family $\left(K\left(X^{\delta}\right)\right)_{\delta>0}$ satisfies $(\star)$ and $(\star \star)$.

Proof of Proposition 4. Notations and first results. Using Convention 2, we also define $S_{s, t}^{\delta}=K_{s, t}\left(X^{\delta}\right)-K_{s, t}^{\delta}(X)$ with

$$
K_{s, t}^{\delta}(X)=\frac{1}{2} \sum_{i=0}^{\ell}\left(Z_{t_{i+1}}+Z_{t_{i}}-2 Z_{s}\right)\left(Y_{t_{i+1}}-Y_{t_{i}}\right) .
$$

From Section 2.3, we know that for any $0 \leq s \leq t \leq 1, K_{s, t}^{\delta}(X)$ converges in probability to $K_{s, t}(X)$. Of course, $K\left(X^{\delta}\right)$ and $K^{\delta}(X)$ will have the same limit.

Lemma 7. The following convergence holds:

$$
\sup _{0 \leq s \leq t \leq t}\left|S_{s, t}^{\delta}\right|=\sup _{0 \leq s \leq t \leq 1}\left|K_{s, t}\left(X^{\delta}\right)-K_{s, t}^{\delta}(X)\right| \underset{\delta \rightarrow 0}{\longrightarrow} 0 \text { a.s.. }
$$

Moreover, $\mathbb{E}\left[\left|S_{s, t}^{\delta}\right|^{\alpha / 2}\right] \preccurlyeq|t-s|^{\alpha / 2}$ for any $0 \leq s \leq t \leq 1$.

Proof. Using Convention 2 (Recall that $t_{0}=s$ and $t_{\ell+1}=t$, which are not necessarily in $\Pi^{\delta}$ ), it follows easily that

$$
S_{s, t}^{\delta}=-\frac{1}{2}\left(Z_{t_{1}}^{\delta}-Z_{t_{0}}^{\delta}\right)\left(Y_{t_{1}}^{\delta}-Y_{t_{0}}^{\delta}\right) \frac{s-t_{0}}{t_{1}-t_{0}}-\frac{1}{2}\left(Z_{t_{\ell+1}}^{\delta}-Z_{t_{\ell}}^{\delta}\right)\left(Y_{t_{\ell+1}}^{\delta}-Y_{t_{\ell}}^{\delta}\right) \frac{t_{\ell+1}-t}{t_{\ell+1}-t_{\ell}} .
$$

Since $X_{t_{1}}^{\delta}=X_{t_{1}}$, it is immediate that

$$
\sup _{0 \leq s \leq t \leq 1}\left|S_{s, t}^{\delta}\right| \preccurlyeq\left\|X-X^{\delta}\right\|_{\infty}\|X\|_{\infty} \underset{\delta \rightarrow 0}{\longrightarrow} 0 \text { a.s. }
$$

which proves the first assertion of the lemma.

Since $\left|t_{\ell+1}-t\right| \leq\left|t_{\ell+1}-t_{\ell}\right|,\left|s-t_{0}\right| \leq\left|t_{1}-t_{0}\right|$, and for any real $a$ and $b$,

$$
|a+b|^{\alpha / 2} \preccurlyeq|a|^{\alpha / 2}+|b|^{\alpha / 2} \text { and }|a b|^{\alpha / 2} \preccurlyeq|a|^{\alpha / 2}+|b|^{\alpha / 2}
$$

the second assertion is also immediate using Lemma 6.

We turn now to the proof of Proposition 4, where we make use of the decomposition of the process $X$ in term of the sum of a forward, a backward and the finite variation term.

We use decomposition (4) with $\varphi(x)=x$ (see Rem. 4), which we rewrite:

$$
\begin{aligned}
& Z_{t}=Z_{0}+\frac{1}{2} M_{t}+\widehat{M}_{t}+V_{t}, \\
& Y_{t}=Y_{0}+\frac{1}{2} N_{t}+\widehat{N}_{t}+W_{t},
\end{aligned}
$$

with

$$
\widehat{M}_{t}=\frac{1}{2}\left(\bar{M}_{1-t}-\bar{M}_{1}\right) \text { and } \widehat{N}_{t}=\frac{1}{2}\left(\bar{N}_{1-t}-\bar{N}_{1}\right)
$$

where $M(\operatorname{resp} . N)$ is the martingale part of $Z$ (resp. $Y$ ), $\bar{M}$ (resp. $\bar{N}$ ) is the martingale part of $Z_{1-t}($ resp. $Y_{1-t}$ ), and $V$ (resp. $W$ ) is the term of finite variation of $Z$ (resp. $Y$ ). Decomposing first $Y$, one gets

$$
K_{s, t}^{\delta}(X)=K_{s, t}^{\delta, 1}(X)+K_{s, t}^{\delta, 2}(X)+T_{s, t}^{\delta}(Z, W)
$$


with

$$
\begin{aligned}
K_{s, t}^{\delta, 1}(X) & =\frac{1}{4} \sum_{i=0}^{\ell}\left(Z_{t_{i+1}}+Z_{t_{i}}-2 Z_{s}\right)\left(N_{t_{i+1}}-N_{t_{i}}\right), \\
K_{s, t}^{\delta, 2}(X) & =\frac{1}{4} \sum_{i=0}^{\ell}\left(Z_{t_{i+1}}+Z_{t_{i}}-2 Z_{s}\right)\left(\widehat{N}_{t_{i+1}}-\widehat{N}_{t_{i}}\right), \\
\text { and } T_{s, t}^{\delta}(Z, W) & =\frac{1}{2} \sum_{i=0}^{\ell}\left(Z_{t_{i+1}}+Z_{t_{i}}-2 Z_{s}\right)\left(W_{t_{i+1}}-W_{t_{i}}\right) .
\end{aligned}
$$

Lemma 8. For any $0 \leq s \leq t \leq 1$, term $T_{s, t}^{\delta}(Z, W)$ converges almost surely to $T_{s, t}(Z, W)=\int_{s}^{t}\left(Z_{r}-Z_{s}\right) \mathrm{d} W_{r}$. If

$$
\widehat{T}_{s, t}(Z, W)=\sup _{r \in[s, t]}\left|Z_{r}-Z_{s}\right| \int_{s}^{t}\left|\mathrm{~d} W_{r}\right|
$$

then $\left|T_{s, t}^{\delta}(Z, W)\right| \leq \widehat{T}_{s, t}(Z, W)$.

Moreover, $\left(T^{\delta}(Z, W)\right)_{\delta>0}$ and $\widehat{T}(Z, W)$ satisfy $(\star)$ and $(\star \star)$.

Proof. The convergence of $T_{s, t}^{\delta}(Z, W)$ to $T_{s, t}(Z, W)$ is immediate, since $W$ is of finite variation.

It is also clear that

$$
\left|T_{s, t}^{\delta}(Z, W)\right| \leq \sup _{r \in[s, t]}\left|Z_{r}-Z_{s}\right| \sum_{i=0}^{\ell}\left|W_{t_{i+1}}-W_{t_{i}}\right| \leq \widehat{T}_{s, t}(Z, W)
$$

for all $(s, t) \in \Delta_{+}$.

We now set

$$
f(r)=\left|b_{j}\left(X_{r}\right)-\frac{a_{j, k}\left(X_{r}\right)}{2 p\left(r, x, X_{r}\right)} \frac{\partial p}{\partial x_{k}}\left(r, x, X_{r}\right)\right| .
$$

We remark that $\sup _{r \in[s, t]}\left|Z_{r}-Z_{s}\right| \leq 2\|Z\|_{\infty}$. For any $\varepsilon>0$, choosing first $\beta>0$ large enough and then $C>0$, one gets from Lemma 1 , Lemma 5 and (5) that

$$
\begin{aligned}
& \mathbb{P}\left[\operatorname{Var}_{\alpha / 2}(\widehat{T}(Z, W)) \geq C\right] \leq \mathbb{P}\left[\operatorname{Var}_{\alpha / 2}(\widehat{T}(Z, W)) \geq C ;\|Z\|_{\infty} \geq \beta\right] \\
& +\mathbb{P}\left[\operatorname{Var}_{\alpha / 2}(\widehat{T}(Z, W)) \geq C ;\|Z\|_{\infty}<\beta\right] \\
& \quad \leq \mathbb{P}\left[\|Z\|_{\infty} \geq \beta\right]+\frac{2 \beta}{C} \mathbb{E}\left[\int_{0}^{1} f(r) \mathrm{d} r\right] \leq \varepsilon .
\end{aligned}
$$

In addition, one may choose $\beta$ and $C$ in function of $\lambda, \Lambda$ and the dimension of the space and then $\widehat{T}_{s, t}(Z, W)$ satisfies $(\star \star)$. Since $\left|T_{s, t}^{\delta}(Z, W)\right| \leq \widehat{T}_{s, t}^{\delta}(Z, W),\left(T^{\delta}(Z, W)\right)_{\delta>0}$ satisfies $(\star \star)$.

Let us denote by $\operatorname{osc}(Z, \eta)$ the modulus of continuity of $Z: \operatorname{osc}(Z, \eta)=\sup _{|t-s| \leq \eta}\left|Z_{t}-Z_{s}\right|$. Then for all $(s, t) \in \Delta_{+}$,

$$
\left|\widehat{T}_{s, t}(Z, W)\right| \leq \operatorname{osc}(Z, t-s) \int_{0}^{1} f(r) \mathrm{d} r
$$


It follows that for any $C>0$ and any $\varepsilon>0$, for any $\beta>0$,

$$
\begin{aligned}
\mathbb{P}\left[\sup _{|t-s|<\eta}\left|\widehat{T}_{s, t}(Z, W)\right| \geq C\right] \leq & \mathbb{P}\left[\operatorname{osc}(Z, \eta) \int_{0}^{1} f(r) \mathrm{d} r \geq C\right] \\
& \leq \mathbb{P}[\operatorname{osc}(Z, \eta) \geq C \varepsilon / \beta]+\mathbb{P}\left[\int_{0}^{1} f(r) \mathrm{d} r \geq \beta / \varepsilon\right] \\
& \leq \frac{\beta^{\alpha} \eta^{\alpha / 2}}{C^{\alpha / 2} \varepsilon^{\alpha}} \mathbb{E}\left[\operatorname{osc}(Z, \eta)^{\alpha}\right]+\frac{\varepsilon}{\beta} \mathbb{E}\left[\int_{0}^{1} f(r) \mathrm{d} r\right] .
\end{aligned}
$$

The last inequality follows from the Bienaymé-Tchebychev inequality and Lemma 3 . With $\beta=\mathbb{E}\left[\int_{0}^{1} f(r) \mathrm{d} r\right]$ and $\eta$ small enough, we get that

$$
\mathbb{P}\left[\sup _{|t-s|<\eta}\left|\widehat{T}_{s, t}(Z, W)\right| \geq C\right] \leq 2 \varepsilon .
$$

Besides, with Lemma 3 and (5), the choice of $\eta$ depends only on $\lambda, \Lambda$ and the dimension of the space. In conclusion, $\widehat{T}(Z, W)$, and thus $\left(T^{\delta}(Z, W)\right)_{\delta>0}$, satisfies $(\star)$.

Proof of Proposition 4. Decomposition of $K^{\delta, 1}(X)$. The term $K_{s, t}^{\delta, 1}(X)$ is itself decomposed as

$$
K_{s, t}^{\delta, 1}(X)=\frac{1}{4} \sum_{i=0}^{\ell}\left(Z_{t_{i+1}}-Z_{t_{i}}\right)\left(N_{t_{i+1}}-N_{t_{i}}\right)+\frac{1}{2} L_{s, t}^{\delta}(Z, N),
$$

with

$$
L_{s, t}^{\delta}(Z, N)=\sum_{i=0}^{\ell}\left(Z_{t_{i}}-Z_{s}\right)\left(N_{t_{i+1}}-N_{t_{i}}\right)
$$

Using the decomposition of $Z$, the term $\sum_{i=0}^{\ell}\left(Z_{t_{i+1}}-Z_{t_{i}}\right)\left(N_{t_{i+1}}-N_{t_{i}}\right)$ is the sum of $\frac{1}{2} Q_{s, t}^{\delta}(M, N)+\frac{1}{2} Q_{s, t}^{\delta}(\bar{M}, N)+$ $Q_{s, t}^{\delta}(V, N)$, where

$$
\begin{aligned}
Q_{s, t}^{\delta}(M, N) & =\sum_{i=0}^{\ell}\left(M_{t_{i+1}}-M_{t_{i}}\right)\left(N_{t_{i+1}}-N_{t_{i}}\right), \\
Q_{s, t}^{\delta}(\widehat{M}, N) & =\sum_{i=0}^{\ell}\left(\widehat{M}_{t_{i+1}}-\widehat{M}_{t_{i}}\right)\left(N_{t_{i+1}}-N_{t_{i}}\right), \\
\text { and } Q_{s, t}^{\delta}(V, N) & =\sum_{i=0}^{\ell}\left(V_{t_{i+1}}-V_{t_{i}}\right)\left(N_{t_{i+1}}-N_{t_{i}}\right) .
\end{aligned}
$$

Lemma 9. The process $\left(L_{s, t}^{\delta}(Z, N)\right)_{0 \leq s \leq t \leq 1}$ defined by (19) satisfies

$$
\mathbb{E}\left[\left|L_{s, t}^{\delta}(Z, N)\right|^{\alpha / 2}\right] \preccurlyeq|t-s|^{\alpha / 2} .
$$


Proof. As $N$ is a $\mathcal{F}_{t}$-martingale which is square-integrable and $Z$ is $\mathcal{F}_{t}$-adapted, thanks to Lemma 3 ,

$$
\begin{aligned}
\mathbb{E}\left[\left|\sum_{i=0}^{\ell}\left(Z_{t_{i}}-Z_{t_{0}}\right)\left(N_{t_{i+1}}-N_{t_{i}}\right)\right|^{\alpha / 2}\right] & \preccurlyeq \mathbb{E}\left[\left|\sum_{i=0}^{\ell}\left(Z_{t_{i}}-Z_{t_{0}}\right)^{2}\left(\langle N\rangle_{t_{i+1}}-\langle N\rangle_{t_{i}}\right)\right|^{\alpha / 4}\right] \\
& \preccurlyeq \mathbb{E}\left[\sup _{s \leq r \leq t}\left|Z_{r}-Z_{s}\right|^{\alpha}\right]+|t-s|^{\alpha / 2} \preccurlyeq|t-s|^{\alpha / 2} .
\end{aligned}
$$

The proof is now complete.

The following Lemma will be useful to deal with $Q^{\delta}(M, N)$ and $Q^{\delta}(\widehat{M}, N)$.

Lemma 10. Let $\delta$ be fixed positive real. Let $M$ be a continuous martingale such that for any $0 \leq s \leq t \leq 1$, $\langle M\rangle_{t}-\langle M\rangle_{s} \preccurlyeq t-s$ almost surely. Then

$$
J_{s, t}^{\delta}(M)=\sum_{i=0}^{\ell}\left(M_{t_{i+1}}-M_{t_{i}}\right)^{2}
$$

satisfies $\mathbb{E}\left[\left|J_{s, t}^{\delta}(M)\right|^{\alpha / 2}\right] \preccurlyeq|t-s|^{\alpha / 2}$.

Proof. By the convexity inequality,

$$
\begin{aligned}
\left|J_{s, t}^{\delta}(M)\right|^{\alpha / 2} & =|t-s|^{\alpha / 2}\left|\sum_{i=0}^{\ell} \frac{t_{i+1}-t_{i}}{t-s} \times \frac{\left(M_{t_{i+1}}-M_{t_{i}}\right)^{2}}{t_{i+1}-t_{i}}\right|^{\alpha / 2} \\
& \leq|t-s|^{\alpha / 2} \sum_{i=0}^{\ell} \frac{t_{i+1}-t_{i}}{t-s} \times \frac{\left|M_{t_{i+1}}-M_{t_{i}}\right|^{\alpha}}{\left|t_{i+1}-t_{i}\right|^{\alpha / 2}} .
\end{aligned}
$$

As $\mathbb{E}\left[\left|M_{t_{i+1}}-M_{t_{i}}\right|^{\alpha}\right] \preccurlyeq \mathbb{E}\left[\left|\langle M\rangle_{t}-\langle M\rangle_{s}\right|^{\alpha / 2}\right] \preccurlyeq|t-s|^{\alpha / 2}$, it is now clear that $\mathbb{E}\left[\left|J_{s, t}^{\delta}(M)\right|^{\alpha / 2}\right] \preccurlyeq \mid t-$ $\left.s\right|^{\alpha / 2}$.

Let us note that

$$
Q_{s, t}^{\delta}(M, N) \leq J_{s, t}^{\delta}(M)+J_{s, t}^{\delta}(N) .
$$

Besides, $\langle M\rangle_{t}-\langle M\rangle_{s}=\int_{s}^{t} a_{i, i}\left(X_{s}\right) \mathrm{d} s,\langle N\rangle_{t}-\langle N\rangle_{s}=\int_{s}^{t} a_{j, j}\left(X_{s}\right) \mathrm{d} s$ and $\langle\bar{M}\rangle_{t}-\langle\bar{M}\rangle_{s}=\int_{1-t}^{1-s} a_{i, i}\left(X_{s}\right) \mathrm{d} s$ and thus,

$$
\langle M\rangle_{t}-\langle M\rangle_{s} \preccurlyeq t-s,\langle N\rangle_{t}-\langle N\rangle_{s} \preccurlyeq t-s,\langle\bar{M}\rangle_{t}-\langle\bar{M}\rangle_{s} \preccurlyeq t-s .
$$

Lemma 10 may then be applied to $J^{\delta}(M)$ and $J^{\delta}(N)$.

We also note that

where $J_{s, t}^{\delta}(V)=\sum_{i=0}^{\ell}\left(V_{t_{i+1}}-V_{t_{i}}\right)^{2}$.

$$
Q_{s, t}^{\delta}(V, N) \leq J_{s, t}^{\delta}(N)+J_{s, t}^{\delta}(V)
$$

Lemma 11. The family $\left(J_{s, t}^{\delta}(V)\right)_{\delta>0}$ converges almost surely to 0 as $\delta \rightarrow 0$ and satisfies

$$
J_{s, t}^{\delta}(V) \leq \widehat{J}_{s, t}(V)=\sup _{r, u \in[s, t]}\left|V_{r}-V_{u}\right| \int_{s}^{t}\left|\mathrm{~d} V_{r}\right| .
$$

In addition, $\widehat{J}(V)$ and $J_{s, t}^{\delta}(V)$ satisfy $(\star)$ and $(\star \star)$. 
Proof. As $V$ is of finite variation, $J^{\delta}(V)$ converges to 0 .

From the Doob inequality and (21), for any $\beta>0$,

$$
\mathbb{P}\left[\|M\|_{\infty} \geq \beta\right] \leq \frac{1}{\beta} \sqrt{\mathbb{E}\left[\langle M\rangle_{1}\right]} \preccurlyeq \frac{1}{\beta} .
$$

Similarly, $\mathbb{P}\left[\|\bar{M}\|_{\infty} \geq \beta\right] \preccurlyeq \beta^{-1}$. In addition, from the Burkholder-Davis-Gundy inequality, (21) and the Kolmogorov criterion (see Lem. 2), it is standard that

$$
\mathbb{E}\left[\sup _{0 \leq s<t \leq 1} \frac{\left|M_{t}-M_{s}\right|}{|t-s|^{1 / \alpha}}\right] \preccurlyeq 1 \text { and } \mathbb{E}\left[\sup _{0 \leq s<t \leq 1} \frac{\left|\bar{M}_{t}-\bar{M}_{s}\right|}{|t-s|^{1 / \alpha}}\right] \preccurlyeq 1 .
$$

It is then possible to estimate $\|V\|_{\infty}$ from $\|Z\|_{\infty},\|M\|_{\infty}$ and $\|\bar{M}\|_{\infty}$, as well as an estimate $\operatorname{osc}(V, \eta)$ from $\operatorname{osc}(Z, \eta), \operatorname{osc}(M, \eta)$, and $\operatorname{osc}(\bar{M}, \eta)$.

The proof is then similar to the one of Lemma 8.

With (20) and (22), it follows that

$$
\left|K_{s, t}^{\delta, 1}(X)\right| \preccurlyeq J_{s, t}^{\delta}(M)+J_{s, t}^{\delta}(N)+J_{s, t}^{\delta}(\bar{M})+\left|L_{s, t}^{\delta}(Z, N)\right|+J_{s, t}^{\delta}(V) .
$$

Proof of Proposition 4. Decomposition of $K^{\delta, 2}(X)$. We rewrite $K^{\delta, 2}(X)$ as

$$
K_{s, t}^{\delta, 2}(X)=\frac{1}{4} \sum_{i=0}^{\ell}\left(\bar{Z}_{1-t_{i+1}}+\bar{Z}_{1-t_{i}}-2 \bar{Z}_{1-t}\right)\left(\bar{N}_{1-t_{i+1}}-\bar{N}_{1-t_{i}}\right)+\frac{1}{2}\left(\bar{Z}_{1-t}-\bar{Z}_{1-s}\right)\left(\bar{N}_{1-t}-\bar{N}_{1-s}\right),
$$

where $\bar{Z}=Z_{1-\text {., }}$ and $\bar{N}$ is the martingale part of $\bar{Y}=Y_{1-.}$.

Let us define $L_{s, t}^{\delta}(\bar{Z}, \bar{N})$ as in (19) with $N$ replaced by $\bar{N}, Z_{t_{i}}$ replaced by $\bar{Z}_{1-t_{i}}$ and $Z_{t_{i}}$ replaced by $\bar{Z}_{1-t_{i}}$. It follows that

$$
\left|K_{s, t}^{\delta, 2}(X)\right| \preccurlyeq J_{s, t}^{\delta}(\bar{M})+J_{s, t}^{\delta}(M)+J_{s, t}^{\delta}(\bar{N})+\left|L_{s, t}^{\delta}(\bar{Z}, \bar{N})\right|+J_{s, t}^{\delta}(V)+\frac{1}{2}\left|\bar{Z}_{1-t}-\bar{Z}_{1-s}\right| \times\left|\bar{N}_{1-t}-\bar{N}_{1-s}\right| .
$$

The conclusions of Lemma 9 are then also true for $L^{\delta}(\bar{Z}, \bar{N})$, since $\bar{N}$ is a martingale with respect to the backward filtration $\left(\overline{\mathcal{F}}_{t}\right)_{t \in[0,1]}$, and $\bar{Z}$ is also adapted to this filtration. Besides, with the Cauchy-Schwarz inequality, the Burkholder-Davis-Gundy inequality and Lemma 3, one gets

$$
\mathbb{E}\left[\left|\bar{Z}_{1-t}-\bar{Z}_{1-s}\right|^{\alpha / 2}\left|\bar{N}_{1-t}-\bar{N}_{1-s}\right|^{\alpha / 2}\right] \preccurlyeq|t-s|^{\alpha / 2}
$$

for all $0 \leq s \leq t \leq 1$.

Proof of Proposition 4. Estimating on the modulus of continuity and the $\alpha / 2$-variation of $K\left(X^{\delta}\right)$. It is now time to gather all our results in order to evaluate the modulus of continuity and the $\alpha$-variation of $K_{s, t}\left(X^{\delta}\right)$.

Up to now, we have bounded $K_{s, t}\left(X^{\delta}\right)$ by the sum of several terms, that can be decomposed in two groups: the first group contains the elements of type $A_{s, t}^{\delta}$ for which $\mathbb{E}\left[\left|A_{s, t}^{\delta}\right|\right] \preccurlyeq|t-s|^{\alpha / 2}$. To this end, we set

$$
\begin{aligned}
\widehat{K}_{s, t}^{\delta}=J_{s, t}^{\delta}(\bar{M})+J_{s, t}^{\delta}(\bar{N})+J_{s, t}^{\delta}(M)+\left|L_{s, t}^{\delta}(\bar{Z}, \bar{N})\right|+J_{s, t}^{\delta}(M)+J_{s, t}^{\delta}(N) & \\
& +J_{s, t}^{\delta}(\widehat{M})+\left|L_{s, t}^{\delta}(Z, N)\right|+\left|S_{s, t}^{\delta}\right|+\frac{1}{2}\left|\bar{Z}_{1-t}-\bar{Z}_{1-s}\right| \times\left|\bar{N}_{1-t}-\bar{N}_{1-s}\right|,
\end{aligned}
$$

which satisfies

$$
\mathbb{E}\left[\left|\widehat{K}_{s, t}^{\delta}\right|^{\alpha / 2}\right] \preccurlyeq|t-s|^{\alpha / 2} .
$$


The second group of terms contains the elements that only satisfy $(\star)$ and $(\star \star)$, that are $J^{\delta}(V)$ and $T^{\delta}(Z, W)$. We set

$$
\widehat{T}_{s, t}=\widehat{T}_{s, t}(Z, W)+\widehat{J}_{s, t}(V)=\sup _{r \in[s, t]}\left|Z_{t}-Z_{s}\right| \int_{s}^{t}\left|\mathrm{~d} W_{r}\right|+\sup _{r, u \in[s, t]}\left|V_{r}-V_{u}\right| \int_{s}^{t}\left|\mathrm{~d} V_{r}\right| .
$$

We have seen in Lemmas 8 and 11 that $\widehat{T}_{\delta>0}$ satisfies $(\star)$ and $(\star \star)$.

We remark also that $\widehat{T}_{s, u}+\widehat{T}_{u, t} \leq \widehat{T}_{s, t}$ for all $0 \leq s \leq u \leq t \leq 1$. Using the decomposition

$$
K_{s, t}\left(X^{\delta}\right)=K_{s, u}\left(X^{\delta}\right)+K_{u, t}\left(X^{\delta}\right)+\left(Z_{u}^{\delta}-Z_{s}^{\delta}\right)\left(Y_{t}^{\delta}-Y_{u}^{\delta}\right)
$$

we get that

for all $0 \leq s \leq u \leq t \leq 1$.

$$
\left|K_{s, t}\left(X^{\delta}\right)\right| \preccurlyeq \widehat{K}_{s, u}^{\delta}+\widehat{K}_{u, t}^{\delta}+\left(Z_{u}^{\delta}-Z_{s}^{\delta}\right)+\left(Y_{t}^{\delta}-Y_{u}^{\delta}\right)+\widehat{T}_{s, t}
$$

The proof is now inspired by the one in [2], where the $\alpha$-variation of a rough path is computed from the dyadics. However, although this method has proved to be successful in various cases, we use it in a slightly different way.

Let $0 \leq s<t \leq 1$ be fixed. For each $n$, let $\Delta^{n}=\left\{i / 2^{n} \mid i=0, \ldots, 2^{n}\right\}$ be sets of dyadics. We set $\eta=t-s$, and let $n(\eta)$ be the smallest integer such that Card $\Delta^{i} \cap[s, t]=2$. Let $t_{-1}<t_{1}$ be the elements in $\Delta^{n(\eta)}$ such that $\left[t_{-1}, t_{1}\right]$ is the largest interval contained in $[s, t]$ among all the intervals of the form $\left[s^{\prime}, t^{\prime}\right]$ with $s^{\prime}, t^{\prime} \in \Delta^{n(\eta)}$. Given some points $t_{-i}$ and $t_{i}$ in $\Delta^{i-1+n(\eta)}$ for some non-negative integer $i$, we construct recursively some new points $t_{-i-1}$ and $t_{i+1}$ in $\Delta^{i+n(\eta)}$ such that $t_{-i-1}=\min \left\{s^{\prime} \in \Delta^{i+n(\eta)} \mid s^{\prime} \geq s\right\}$ and $t_{i+1}=\max \left\{t^{\prime} \in \Delta^{i+n(\eta)} \mid t^{\prime} \leq t\right\}$. Our construction ensures that $[s, t]=\cdots \cup\left[t_{-3}, t_{-2}\right] \cup\left[t_{-2}, t_{-1}\right] \cup\left[t_{-1}, t_{1}\right] \cup$ $\left[t_{1}, t_{2}\right] \cup\left[t_{2}, t_{3}\right] \cup \cdots$. Moreover, when $\left[t_{i}, t_{i+1}\right]$ is not reduced to a singleton, then there exist some integers $k$ and $n$ such that $t_{i}=k / 2^{n}$ and $t_{i+1}=(k+1) / 2^{n}$. The number of intervals to consider may be countable. With (24) the continuity of $K\left(X^{\delta}\right)$ and (25),

$$
\begin{aligned}
\left|K_{s, t}\left(X^{\delta}\right)\right| & \leq \sum_{i=-\infty}^{+\infty}\left|K_{t_{i}, t_{i+1}}\left(X^{\delta}\right)\right|+\sum_{i, j=-\infty}^{+\infty}\left|Z_{t_{i+1}}^{\delta}-Z_{t_{i}}^{\delta}\right| \times\left|Y_{t_{j+1}}^{\delta}-Y_{t_{j}}^{\delta}\right| \\
& \leq L_{s, t}^{\delta}+\widehat{T}_{s, t} .
\end{aligned}
$$

with the convention that $t_{0}=t_{1}$ and

$$
L_{s, t}^{\delta}=\sum_{i=-\infty}^{+\infty}\left|\widehat{K}_{t_{i}, t_{i+1}}^{\delta}\right|+\sum_{i, j=-\infty}^{+\infty}\left|Z_{t_{i+1}}^{\delta}-Z_{t_{i}}^{\delta}\right| \times\left|Y_{t_{j+1}}^{\delta}-Y_{t_{j}}^{\delta}\right| .
$$

We now focus on $\left(L^{\delta}\right)_{\delta>0}$, in order to prove that it satisfies $(\star)$ and $(\star \star)$. This will then prove that $\left(K\left(X^{\delta}\right)\right)_{\delta>0}$ also satisfies $(\star)$ and $(\star \star)$, since from Lemmas 8 and 11, $\widehat{T}$ satisfies $(\star)$ and $(\star \star)$.

Let $\alpha>2$ and $c_{i}=(|i|+1+n(\eta))^{\alpha / 2}$. Hence, $C_{\alpha}=\left(\sum_{i=-\infty}^{+\infty} 1 / c_{i}^{\alpha /(\alpha-2)}\right)^{\alpha / 2-1}$ is finite. By application of the Hölder inequality,

$$
\left|L_{s, t}^{\delta}\right|^{\alpha / 2} \leq\left(3^{\alpha}-1\right) C_{\alpha} \sum_{i=-\infty}^{\infty}(1+|i|+n(\eta))^{\alpha^{2} / 4}\left(\left|\widehat{K}_{t_{i}, t_{i+1}}^{\delta}\right|{ }^{\alpha / 2}+2^{\alpha-1}\left|Z_{t_{i+1}}^{\delta}-Z_{t_{i}}^{\delta}\right|^{\alpha}+2^{\alpha-1}\left|Y_{t_{i+i}}^{\delta}-Y_{t_{i}}^{\delta}\right|^{\alpha}\right) .
$$

We are then led to the following inequality:

$$
\begin{aligned}
\left|L_{s, t}^{\delta}\right|^{\alpha / 2} \preccurlyeq \sum_{n \geq n(\eta)}(1+n)^{\alpha^{2} / 4} \sum_{i=0, \ldots, 2^{n}-1, i / 2^{n} \in[s, t]}\left(\left|\widehat{K}_{i / 2^{n},(i+1) / 2^{n}}^{\delta}\right|^{\alpha / 2}\right. & \\
& \left.+\left|Z_{(i+1) / 2^{n}}^{\delta}-Z_{i / 2^{n}}^{\delta}\right|^{\alpha}+\left|Y_{(i+1) / 2^{n}}^{\delta}-Y_{i / 2^{n}}^{\delta}\right|^{\alpha}\right) .
\end{aligned}
$$


Setting

$$
C_{n}=\sum_{i=0}^{2^{n}-1}\left(\left|\widehat{K}_{i / 2^{n},(i+1) / 2^{n}}\left(X^{\delta}\right)\right|^{\alpha / 2}+\left|Z_{(i+1) / 2^{n}}^{\delta}-Z_{i / 2^{n}}^{\delta}\right|^{\alpha}+\left|Y_{(i+1) / 2^{n}}^{\delta}-Y_{i / 2^{n}}^{\delta}\right|^{\alpha}\right),
$$

we deduce from Lemma 3, (23) and (26) that

$$
\operatorname{Var}_{\alpha / 2}\left(L^{\delta}\right)^{\alpha / 2} \preccurlyeq \sum_{n \geq 0}(1+n)^{\alpha^{2}} C_{n} .
$$

With (23),

$$
\mathbb{E}\left[\sum_{i=0}^{2^{n}-1}\left|\widehat{K}_{i / 2^{n},(i+1) / 2^{n}}^{\delta}\right|^{\alpha / 2}\right] \leq 2^{n}\left(\frac{1}{2^{n}}\right)^{\alpha / 2} \leq 2^{n(1-\alpha / 2)} .
$$

From Lemma 3 and the proof of Lemma 6 , it is easily seen that $\mathbb{E}\left[\left|Z_{t}^{\delta}-Z_{s}^{\delta}\right|^{\alpha}\right] \preccurlyeq|t-s|^{\alpha / 2}$ and $\mathbb{E}\left[\left|Y_{t}^{\delta}-Y_{s}^{\delta}\right|^{\alpha}\right] \preccurlyeq$ $|t-s|^{\alpha / 2}$ for any $0 \leq s \leq t \leq 1$. Thus, an inequality similar to $(28)$ holds when $\left|\widehat{K}_{s, t}\left(X^{\delta}\right)\right|^{\alpha / 2}$ is replaced by $\left|Y_{t}^{\delta}-Y_{s}^{\delta}\right|^{\alpha}$ and $\left|Z_{t}^{\delta}-Z_{s}^{\delta}\right|^{\alpha}$.

As $\alpha>2$, the series $\sum_{n \geq 0}(1+n)^{\alpha^{2}} 2^{n(1-\alpha / 2)}$ converges. From Inequality $(27), \sup _{\delta>0} \mathbb{E}\left[\operatorname{Var}_{\alpha / 2} L^{\delta}\right] \preccurlyeq 1$ and then $\left(L^{\delta}\right)_{\delta>0}$ satisfies $(\star \star)$.

To prove that $\left(L^{\delta}\right)_{\delta>0}$ satisfies $(\star)$, we use $(26)$ as previously to deduce that

$$
\sup _{|t-s|<\eta}\left|L_{s, t}^{\delta}\right|^{\alpha / 2} \preccurlyeq \sum_{n \geq n(\eta)}(1+n)^{\alpha^{2}} C_{n} .
$$

The difference with (27) lies in the fact that the sum is taken for dyadic starting at size $1 / 2^{n(\eta)}$. However, we have seen that $\sum_{n \geq 0}(1+n)^{\alpha^{2}} \mathbb{E}\left[C_{n}\right] \preccurlyeq 1$. Since $n(\eta)$ is deterministic and $n(\eta)$ increases to $+\infty$ as $\eta$ goes to 0

$$
\sup _{\delta>0} \mathbb{E}\left[\sup _{|t-s|<\eta}\left|L_{s, t}^{\delta}\right|^{\alpha / 2}\right] \underset{\varepsilon \rightarrow 0}{\longrightarrow} 0 .
$$

Hence, $\left(L^{\delta}\right)_{\delta>0}$ satisfies $(\star)$.

This concludes the proof of Proposition 4.

\subsection{Convergence of the brackets}

Combined with Theorem 1, the convergence of $K\left(X^{\delta}\right)$ leads to the construction of $K(X)$ and to integrals of Stratonovich type. To construct an integral of Itô type, we need to control also the brackets of $X$.

We have seen in Section 2.3 that

$$
\int_{s}^{t}\left(Z_{r}-Z_{s}\right) \circ \mathrm{d} Y_{r}=\int_{s}^{t}\left(Z_{r}-Z_{s}\right) \mathrm{d} Y_{r}+\frac{1}{2}\left(\langle Z, Y\rangle_{t}-\langle Z, Y\rangle_{s}\right)
$$

and that, in probability,

$$
\langle Z, Y\rangle_{t}=\lim _{\delta \rightarrow 0} Q_{t}^{\delta}
$$

where $Q^{\delta}=Q^{\delta, i, j}$ is defined by (10) with $Z=X^{i}$ and $Y=X^{j}$, and where the indices $i$ and $j$ have been dropped. Note that since $X$ is a Dirichlet process, $\langle Z, Y\rangle$ is also equal to the brackets of the martingale parts $M$ and $N$ of $Z$ and $Y$. 
Lemma 12. For any $\eta>0$,

Besides, for any $\alpha>2, \sup _{\delta>0} \mathbb{E}\left[\operatorname{Var}_{\alpha / 2} Q^{\delta}\right] \preccurlyeq 1$.

$$
\sup _{\delta>0} \mathbb{E}\left[\sup _{|t-s|<\eta}\left|Q_{t}^{\delta}-Q_{s}^{\delta}\right|\right] \preccurlyeq \eta^{1 / \alpha}
$$

Proof. Let us denote by $\left(J_{s, t}^{\delta}(X)\right)_{0 \leq s \leq t \leq 1}$ the process

$$
J_{s, t}^{\delta}(X)=\sum_{i=0}^{\ell}\left|X_{t_{i+1}}-X_{t_{i}}\right|^{2}
$$

We will show that $J^{\delta}(X)$ satisfies $\mathbb{E}\left[\operatorname{Var}_{\alpha / 2} J^{\delta}(X)\right] \preccurlyeq 1$ for any $\alpha>2$ and $\mathbb{E}\left[\sup _{|t-s|<\eta} J_{s, t}^{\delta}(X)\right] \preccurlyeq \eta$. By the Fatou lemma, this clearly implies the conclusions of Lemma 12.

For any $t \in[0,1]$, let $L^{\delta}$ denotes the continuous function

$$
L_{t}^{\delta}=\sum_{i=0}^{\ell^{\prime}-1}\left|X_{t_{i+1}^{\prime}}-X_{t_{i}^{\prime}}\right|^{2}+\frac{t-t_{\ell^{\prime}}^{\prime}}{t_{\ell^{\prime}+2}^{\prime}-t_{\ell^{\prime}}^{\prime}}\left|X_{t_{\ell^{\prime}+2}^{\prime}}-X_{t_{\ell^{\prime}}^{\prime}}\right|^{2}
$$

where $t_{0}^{\prime} \leq \ldots \leq t_{\ell^{\prime}}^{\prime}$ are the points of $\Pi^{\delta} \cap[0, t]$ and $t_{\ell^{\prime}+2}$ is the point in $\Pi^{\delta}$ and not in $[0, t)$ closest to $t$. So, with Convention 2 in force,

$$
L_{t}^{\delta}-L_{s}^{\delta}=\sum_{i=1}^{\ell-1}\left|X_{t_{i+1}}-X_{t_{i}}\right|^{2}+\frac{t_{1}-s}{t_{1}-t_{-1}}\left|X_{t_{1}}-X_{t_{-1}}\right|^{2}+\frac{t-t_{\ell}}{t_{\ell+2}-t_{\ell}}\left|X_{t_{\ell+2}}-X_{t_{\ell}}\right|^{2}
$$

Using the convexity inequality as in the proof of Lemma 10,

$$
\left|\sum_{i=1}^{\ell-1}\right| X_{t_{i+1}}-\left.\left.X_{t_{i}}\right|^{2}\right|^{\alpha / 2} \preccurlyeq\left|t_{\ell}-t_{1}\right|^{\alpha / 2}
$$

Furthermore,

$$
\mathbb{E}\left[\left(\frac{t-t_{\ell}}{t_{\ell+2}-t_{\ell}}\left|X_{t_{\ell+2}}-X_{t_{\ell}}\right|^{2}\right)^{\alpha / 2}\right] \preccurlyeq\left|t-t_{\ell}\right|^{\alpha / 2},
$$

and a similar relation holds for $\frac{t_{1}-s}{t_{1}-t_{-1}}\left|X_{t_{1}}-X_{t_{-1}}\right|^{2}$. So, it is easily deduced that

$$
\mathbb{E}\left[\left|L_{t}^{\delta}-L_{s}^{\delta}\right|^{\alpha / 2}\right] \preccurlyeq|t-s|^{\alpha / 2} \text { and } \mathbb{E}\left[\operatorname{Var}_{\alpha / 2} L^{\delta}\right] \preccurlyeq 1 \text {. }
$$

The functions $J^{\delta}(X)$ and $L^{\delta}$ satisfy

$$
J_{s, t}^{\delta}(X)=L_{t}^{\delta}-L_{s}^{\delta}-\frac{t_{1}-s}{t_{1}-t_{-1}}\left|X_{t_{1}}-X_{t_{-1}}\right|^{2}+\left|X_{t_{1}}-X_{s}\right|^{2}-\frac{t-t_{\ell}}{t_{\ell+2}-t_{\ell}}\left|X_{t_{\ell+2}}-X_{t_{\ell}}\right|^{2}+\left|X_{t_{\ell+1}}-X_{t_{\ell}}\right|^{2}
$$

But $\left(t-t_{\ell}\right) /\left(t_{\ell+2}-t_{\ell}\right) \leq 1$ and $\left(t_{1}-s\right) /\left(t_{1}-t_{-1}\right) \leq 1$. Furthermore, for any partition $\Pi^{\delta}, \sum_{\Pi^{\delta}}\left|X_{t_{\ell+2}}-X_{t_{\ell}}\right|^{\alpha} \leq$ $\operatorname{Var}_{\alpha} X$ and $\sum_{\Pi^{\delta}}\left|X_{t_{\ell+1}}-X_{t_{\ell}}\right|^{\alpha} \leq \operatorname{Var}_{\alpha} X$ (recall that by Convention 2, $t_{\ell}$ and $t_{\ell+2}$ depend on the points in $\left.\Pi^{\delta}\right)$. So, $\operatorname{Var}_{\alpha / 2}\left(J^{\delta}(X)\right)^{\alpha} \preccurlyeq \operatorname{Var}_{\alpha / 2}(L)^{\alpha / 2}+\operatorname{Var}_{\alpha}(X)^{\alpha}$.

Besides, from the Kolmogorov Lemma 2, we know that for any $\beta<\alpha^{-1}(\alpha / 2-1)$, there exists some nonnegative random variable $C_{\beta}(\omega)$ such that $\left|L_{t}^{\delta}-L_{s}^{\delta}\right|^{\alpha / 2}+\left|X_{t}-X_{s}\right|^{\alpha}<C_{\beta}(\omega)|t-s|^{\alpha \beta}$ and $\mathbb{E}\left[C_{\beta}(\omega)\right] \preccurlyeq 1$. Hence, choosing $\beta$ so that $\beta \alpha=1, \mathbb{E}\left[\sup _{|t-s|<\eta}\left|J_{s, t}^{\delta}(X)\right|\right] \preccurlyeq \eta^{1 / \alpha}$. 
The lemma is proved by combining the previous estimates on $L^{\delta}$ and $J^{\delta}(X)$, since from the Cauchy-Schwarz inequality, $\left|Q_{t}^{\delta}-Q_{s}^{\delta}\right| \leq L_{t}^{\delta}-L_{s}^{\delta}$.

\subsection{Proof of Theorem 2}

Theorem 2 is now easily proved from Proposition 4 and Lemma 12.

Proof of Theorem 2. With Corollary 3 and Lemma 4, that $\left(K\left(X^{\delta}\right)\right)_{\delta>0}$ satisfies $(\star)$ and $(\star \star)$ is sufficient to assert that the sequence $\left(X^{\delta}, K\left(X^{\delta}\right)\right)_{\delta>0}$ is tight in $\mathcal{V}^{\alpha}$. Let $\mathbf{X}=\left(\mathbf{X}^{1}, \mathbf{X}^{2}\right)$ be one of its limits. By identification, $\mathbf{X}_{s, t}^{1}=X_{t}-X_{s}$ for any $0 \leq s \leq t \leq 1$, since $X^{\delta}$ converges uniformly to $X$.

It remains to identify $\mathbf{X}^{2}$ with $K(X)$.

We have seen in Section 2.3 that for any $(s, t) \in \Delta_{+}, K_{s, t}^{\delta}(X)$ converges in probability to $K_{s, t}(X)$ for any $(s, t) \in \Delta_{+}$. Since $\left(K\left(X^{\delta}\right)\right)_{\delta>0}$ satisfies $(\star)$, Lemma 7 , the continuity of $X,(17)$ and (18) are sufficient to prove that $t \mapsto K_{0, t}^{\delta}(X)$ is continuous and that $\left(t \mapsto K_{0, t}^{\delta}(X)\right)_{\delta>0}$ is tight in the space of continuous functions, and

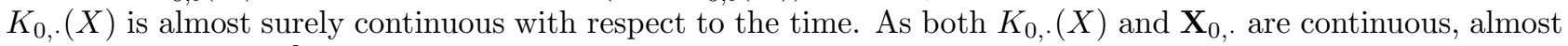
surely, $K_{s, t}(X)=\mathbf{X}_{s, t}^{2}$ for any $(s, t) \in \Delta_{+}$.

With Lemma 12 and (29), it is easily shown that $K^{\text {ito }}(X)$ is of finite $\alpha / 2$-variation for all $\alpha>1$, and that $K^{\text {itô }}\left(X^{\delta}\right)$ converges $K^{\text {itô }}(X)$ in $\alpha / 2$-variation as we did for the Stratonovich integrals.

Point (iii) follows directly from Proposition 3.

\section{Construction of different rough paths lying ABove the same process}

\subsection{Different areas lead to different integrals}

We have constructed a process $\left(K_{s, t}(X)\right)_{0 \leq s}$ such that $\mathbf{X}=(X, K(X))$ is a geometric rough path. Thus, $K(X)$ appears to be the "natural choice" for constructing a rough path lying above $X$. However, there are other rough paths lying above the same process $X$. In fact (see [24], Lem. 2.2.3, p. 250), if $\mathbf{X}=(X, \widehat{K}(X))$ is another rough path of finite $\alpha$-variation, then there exists some function $\varphi=\left(\varphi_{i, j}\right)_{i, j=1}^{N}$ of finite $\alpha / 2$-variation on $[0,1]$ such that

$$
\widehat{K}_{s, t}^{i, j}(X)=K_{s, t}^{i, j}(X)+\varphi_{i, j}(t)-\varphi_{i, j}(s) .
$$

Conversely, given such a function $\varphi$, then $\widehat{\mathbf{X}}=(X, \widehat{K}(X))$ with $\widehat{K}_{s, t}(X)=K_{s, t}(X)+\varphi(t)-\varphi(s)$ is also a rough path. Moreover, if $\varphi$ is anti-symmetric, i.e., $\varphi_{i, j}(t)=-\varphi_{j, i}(t)$ for any $t \in[0,1]$ and $\mathbf{X}$ is a geometric rough path, then $\widehat{\mathbf{X}}$ is also a geometric rough path. From Proposition 3 , there exists a sequence $\left(\widehat{\mathbf{X}}^{\delta}\right)_{\delta>0}$ of geometric rough paths lying above a piecewise smooth path $\widehat{X}^{\delta}$ which converges to $\widehat{\mathbf{X}}$ in $\mathcal{V}^{\alpha^{\prime}}$ for all $\alpha^{\prime}>\alpha$.

Using the way rough paths are constructed, we obtain easily the following results (see [20] for details). We assume that $\varphi$ is of $\alpha / 2$-finite-variation for any $\alpha>2$, and that $\widehat{\mathbf{X}}$ is the geometric rough path constructed as above.

We write abusively $Y_{t}=Y_{0}+\int_{0}^{t} f\left(Y_{s}\right) \mathrm{d} \mathbf{X}_{s}$ to denote $\mathbf{Y}_{0, t}^{1}+Y_{0}$, where $\mathbf{Y}=Y_{0}+\int_{0}^{\cdot} f\left(\mathbf{Y}_{0, s}\right) \mathrm{d} \mathbf{X}_{s}$.

Let $f$ be a bounded, continuous function with a bounded derivative which is also $\beta$-Hölder continuous for some $\beta>0$. Then

$$
\int_{0}^{t} f\left(X_{r}\right) \mathrm{d} \widehat{\mathbf{X}}_{r}=\int_{0}^{t} f\left(X_{r}\right) \mathrm{d} \mathbf{X}_{r}+\frac{1}{2} \sum_{i, j=1}^{N} \int_{0}^{t}\left(\frac{\partial f^{i}}{\partial x_{j}}-\frac{\partial f^{j}}{\partial x_{i}}\right)\left(X_{r}\right) \mathrm{d} \varphi_{i . j}(r) .
$$

Concerning SDEs, if $f: \mathbb{R}^{m} \rightarrow \mathbb{R}^{m}$ is a bounded, continuous function with two first bounded and continuous derivatives, and such that $\frac{\partial^{2} f}{\partial x_{i} \partial x_{j}}$ is $\beta$-Hölder continuous for some $\beta>0$. Then the solution $Y$ of

$$
Y_{t}=Y_{0}+\int_{0}^{t} f\left(Y_{s}\right) \mathrm{d} \widehat{\mathbf{X}}_{s}
$$


is also solution to

$$
Y_{t}=Y_{0}+\int_{0}^{t} f\left(Y_{s}\right) \mathrm{d} \mathbf{X}_{s}+\sum_{i, j=1}^{N} \int_{0}^{t} \frac{1}{2}\left[f^{i}, f^{j}\right]\left(Y_{s}\right) \mathrm{d} \varphi_{i, j}(s),
$$

where $\left[f^{i}, f^{j}\right]$ is the Lie bracket of the vector fields $f^{i}$ and $f^{j}$, i.e., $\left[f^{i}, f^{j}\right]=\left(\sum_{k=1}^{N} f_{k}^{i} \frac{\partial f_{\ell}^{j}}{\partial x_{k}}-f_{k}^{j} \frac{\partial f_{\ell}^{i}}{\partial x_{k}}\right)_{\ell=1, \ldots, m}$. Thus, these results are an extension of the theorems on the addition of a corrective drift in the Wong-Zakai theorem, that are initially due to McShane [27]: see for example Section VI-7 in [14] of Chapter 5.7 in [15], p. 274, for some account in the Brownian case.

\subsection{An explicit construction by the choice of a good interpolation}

In this section, we show how to construct a rough path as limit of smooth rough path, but with a different area. This construction is similar to the one given for the Brownian motion [20] and leads to the same kind of results.

We assume that the dimension of the space $N$ is equal to 2 . Let $c$ be a real number. Let $\varphi:[0,1] \mapsto \mathbb{R}^{2}$ be a continuous, smooth function such that $\varphi(0)=(0,0), \varphi(1)=(1,0)$ and

$$
c=\frac{1}{2} \int_{0}^{1} \varphi_{1}(s) \varphi_{2}^{\prime}(s) \mathrm{d} s-\frac{1}{2} \int_{0}^{1} \varphi_{2}(s) \varphi_{1}^{\prime}(s) \mathrm{d} s,
$$

with $\varphi=\left(\varphi_{1}, \varphi_{2}\right)$.

Given a trajectory $t \mapsto X_{t}(\omega)$ of the process $X$, we use the function $\varphi$, after a proper scale change and a rotation, to join the sample points $X_{s_{i}}$ and $X_{s_{i+1}}$ for $\Pi^{\delta}=\left\{s_{i} \mid 0 \leq s_{1} \leq s_{2} \leq \cdots \leq s_{k} \leq t\right\}$ : In the complex plane (with $\mathrm{j}^{2}=-1$ ),

$$
\widehat{X}_{t}^{1, \delta}+\mathrm{j} \widehat{X}_{t}^{2, \delta}=X_{s_{i}}^{1}+\mathrm{j} X_{s_{i}}^{2}+\left(\varphi_{1}+\mathrm{j} \varphi_{2}\right) \frac{t-s_{i}}{s_{i+1}-s_{i}}\left(X_{s_{i+1}}^{1}+\mathrm{j} X_{s_{i}}^{2}-X_{s_{i+1}}^{1}-\mathrm{j} X_{s_{i+1}}^{2}\right)
$$

for $t \in\left[s_{i}, s_{i+1}\right]$. We assume that the mesh of $\Pi^{\delta}$ decreases to zero as $\delta$ decreases to 0 .

If $\varphi(t)=(t, 0)$, then $\widehat{X}^{\delta}(\omega)=X^{\delta}(\omega)$, where $X^{\delta}(\omega)$ is the piecewise linear approximation of $X$ along $\left(\Pi^{\delta}\right)_{\delta>0}$ given by (14). It is clear that $\widehat{X}^{\delta}$ converges almost surely to $X$ in $\alpha$-variation. We study now the influence of the choice of $\varphi$ on the limit on $K\left(X^{\varepsilon}\right)$.

For a path $Y$ in $\mathbb{R}^{2}$, we set

$$
A_{s, t}(Y)=\frac{1}{2}\left(K_{s, t}^{1,2}(Y)-K_{s, t}^{2,1}(Y)\right)
$$

Then $A_{s, t}(Y)$ corresponds to the area enclosed between the curve $r \in[s, t] \mapsto Y_{r}$ and the chord $\overline{Y_{s} Y_{t}}$. Clearly, for $\widehat{X}^{\delta}$, this area is

with

$$
A_{s, t}\left(\widehat{X}^{\delta}\right)=A_{s, t}\left(X^{\delta}\right)+A_{s, t}^{\operatorname{corr}}\left(X^{\delta}\right)
$$

$$
A_{s, t}^{\mathrm{corr}}\left(X^{\delta}\right)=\sum_{i=1}^{\ell-1} c\left|\Delta_{i} X\right|^{2}+A_{s, t}^{\mathrm{corr}, \text { left }}+A_{s, t}^{\mathrm{corr}, \text { right }}
$$

where $\Delta_{i} X=X_{t_{i+1}}-X_{t_{i}}$, and the terms $A_{s, t}^{\text {corr, left }}$ and $A_{s, t}^{\text {corr, right }}$ are the areas added by $\varphi$ between times $s$ and $t_{1}$, and $t_{\ell}$ and $t$.

Proposition 5. The corrective term $A^{\mathrm{corr}}\left(X^{\delta}\right)$ converges in probability in $\alpha / 2$-variation to $c Q(X)$ with

$$
Q(X):(s, t) \mapsto\left(\langle M\rangle_{t}-\langle M\rangle_{s}+\langle N\rangle_{t}-\langle N\rangle_{s}\right)
$$

where $M$ and $N$ denotes the martingale parts of $Z$ and $Y$. 
Proof. The supplementary areas $A_{s, t}^{\text {corr, left }}$ and $A_{s, t}^{\text {corr, right }}$ between times $s$ and $t_{1}$ and times $t_{\ell}$ and $t$ are respectively bounded by $c\left|X_{t_{1}}-X_{s}\right|^{2}$ and by $c\left|X_{t}-X_{t_{\ell}}\right|^{2}$. These two terms decrease to 0 , and have integrable $\alpha / 2$-variation and an integrable modulus of continuity.

So, we are interested in studying the limit of $\sum_{i=1}^{\ell-1} c\left|X_{t_{i+1}}-X_{t_{i}}\right|^{2}$. The process $X=(Z, Y)$ is a Dirichlet process with decomposition $X=X_{0}+(M+A, N+B)$. But we know that for any $0 \leq s \leq t \leq 1$

$$
\sum_{i=0}^{\ell}\left|\left(M_{t_{i+1}}-M_{t_{i}}, N_{t_{i+1}}-N_{t_{i}}\right)\right|^{2}-\left(\langle M\rangle_{t}-\langle M\rangle_{s}+\langle N\rangle_{t}-\langle N\rangle_{s}\right) \underset{\delta \rightarrow 0}{\stackrel{\text { probab. }}{\longrightarrow} 0}
$$

and that

$$
\sum_{i=0}^{\ell}\left(\left(A_{t_{i+1}}-A_{t_{i}}\right)^{2}+\left(B_{t_{i+1}}-B_{t_{i}}\right)^{2}\right) \underset{\delta \rightarrow 0}{\stackrel{\text { probab. }}{\longrightarrow}} 0 .
$$

This proposition is now easily proved with Lemma 12.

Corollary 4. We use the notations previously introduced. For any $\alpha>2, \widehat{\mathbf{X}}^{\delta}=\left(\widehat{X}^{\delta}, K\left(\widehat{X}^{\delta}\right)\right)$ converges in $\mathcal{V}^{\alpha}$ to $\widehat{\mathbf{X}}=(X, K(X)+\widehat{c} Q(X))$, where $\widehat{c}$ is the anti-symmetric matrix defined by $\widehat{c}_{1,2}=-\widehat{c}_{1,2}=c$.

Acknowledgements. This work was started while the author was a post-doctoral student in Oxford and granted by the TMR Stochastic Analysis Network. Thus, the author wishes to thank Prof. T.J. Lyons for his kind hospitality and for having learned him the theory of rough paths.

\section{REFERENCES}

[1] D.G. Aronson, Non-negative solutions of linear parabolic equation. Ann. Scuola Norm. Sup. Pisa 22 (1968) 607-693.

[2] R.F. Bass, B. Hambly and T.J. Lyons, Extending the Wong-Zakai theorem to reversible Markov processes. J. Eur. Math. Soc. 4 (2002) 237-269.

[3] K.-T. Chen, Integration of paths, geometric invariants and a generalized Baker-Hausdorff formula. Ann. of Math. 65 (1957) 163-178.

[4] L. Coutin and A. Lejay, Semi-martingales and rough paths theory. Electron. J. Probab. 10 (2005) 761-785.

[5] L. Coutin and Z. Qian, Stochastic analysis, rough path analysis and fractional Brownian motions. Probab. Theory Related Fields 122 (2002) 108-140.

[6] F. Coquet and L. Słomiński, On the convergence of Dirichlet processes. Bernoulli 5 (1999) 615-639.

[7] K. Dupoiron, P. Mathieu and J. San martin, Formule d'Itô pour des diffusions uniformément elliptiques et processus de Dirichlet. Potential Anal. 21 (2004) 7-3.

[8] H. Föllmer, Calcul d'Itô sans probabilités, in Séminaire de Probabilités, XV. Lect. Notes Math. 850 (1981) 143-150. Springer, Berlin.

[9] H. Föllmer, Dirichlet processes, in Stochastic integrals (Proc. Sympos., Univ. Durham, Durham, 1980). Lect. Notes Math. 851 (1981) 476-478. Springer, Berlin.

[10] M. Fukushima, Y. Oshima and M. Takeda, Dirichlet Forms and Symmetric Markov Process. De Gruyter (1994).

[11] F. Flandoli and F. Russo, Generalized integration and stochastic ODEs. Ann. Probab. 30 (2002) 270-292.

[12] P. Friz and N. Victoir, A note on the notion of geometric rough paths. To appear in Probab. Theory Related Fields (2006).

[13] B.M. Hambly and T.J. Lyons, Stochastic area for Brownian motion on the Sierpinski gasket. Ann. Probab. 26 (1998) $132-148$.

[14] N. Ikeda and S. Watanabe, Stochastic Differential Equations and Diffusion Processes. North Holland, 2nd edition (1989).

[15] H. Kunita, Stochastic flows and stochastic differential equations. Cambridge University Press (1990).

[16] A. Lejay, Méthodes probabilistes pour l'homogénéisation des opérateurs sous forme-divergence : cas linéaires et semi-linéaires. Ph.D. thesis, Université de Provence, Marseille, France (2000). www.iecn.u-nancy.fr/ lejay/.

[17] A. Lejay, An introduction to rough paths, in Séminaire de probabilités, XXXVII. Lect. Notes Math. 1832 (2003) 1-59, Springer, Berlin.

[18] A. Lejay, A Probabilistic Representation of the Solution of some Quasi-Linear PDE with a Divergence-Form Operator. Application to Existence of Weak Solutions of FBSDE. Stochastic Process. Appl. 110 (2004) 145-176.

[19] A. Lejay, Stochastic Differential Equations driven by processes generated by divergence form operators II: Convergence results. Institut Élie Cartan de Nancy (preprint), 2003. 
[20] A. Lejay and T.J. Lyons, On the Importance of the Lévy Area for Systems Controlled by Converging Stochastic Processes. Application to Homogenization, in New Trend in Potential Theory, D. Bakry, L. Beznea, Gh. Bucur and M. Röckner Eds., The Theta Foundation (2006).

[21] M. Ledoux, T. Lyons and Z. Qian, Lévy area of Wiener processes in Banach spaces. Ann. Probab. 30 (2002) 546-578.

[22] T. Lyons and Z. Qian, System Control and Rough Paths. Oxford Mathematical Monographs. Oxford University Press (2002).

[23] T.J. Lyons and L. Stoica, The limits of stochastic integrals of differential forms. Ann. Probab. 27 (1999) 1-49.

[24] T.J. Lyons, Differential equations driven by rough signals. Rev. Mat. Iberoamericana 14 (1998) 215-310.

[25] A. Lejay and N. Victoir, On ( $p, q)$-rough paths. J. Differential Equations 225 (2006) 103-133.

[26] Z. Ma and M. Röckner, Introduction to the Theory of (Non-Symmetric) Dirichlet Forms. Universitext. Springer-Verlag (1991).

[27] E.J. McShane. Stochastic differential equations and models of random processes. In Proceedings of the Sixth Berkeley Symposium on Mathematical Statistics and Probability (Univ. California, Berkeley, Calif., 1970/1971), Vol. III: Probability theory, pp. 263-294. Univ. California Press (1972).

[28] A. Rozkosz, Stochastic Representation of Diffusions Corresponding to Divergence Form Operators. Stochastic Process. Appl. 63 (1996) 11-33.

[29] A. Rozkosz, On Dirichlet processes associated with second order divergence form operators. Potential Anal. 14 (2001) $123-148$.

[30] A. Rozkosz and L. Slomiński, Extended Convergence of Dirichlet Processes. Stochastics Stochastics Rep. 65 (1998) $79-109$.

[31] D. Revuz and M. Yor, Continuous Martingales and Brownian Motion. Springer-Verlag (1990).

[32] E.-M. Sipiläinen, A pathwise view of solutions of stochastic differential equations. Ph.D. thesis, University of Edinburgh (1993).

[33] D.W. Stroock, Diffusion Semigroups Corresponding to Uniformly Elliptic Divergence Form Operator, in Séminaire de Probabilités XXII. Lect. Notes Math. 1321 (1988) 316-347. Springer-Verlag.

[34] D.R.E. Williams, Path-wise solutions of SDE's driven by Lévy processes. Rev. Mat. Iberoamericana 17 (2002) $295-330$. arXiv:math.PR/0001018.

[35] E. Wong and M. Zakai, On the convergence of ordinary integrals to stochastic integrals. Ann. Math. Statist. 36 (1965) 15601564 . 